Pacific Journal of Mathematics

EXTENSIONS OF PRO-AFFINE ALGEBRAIC GROUPS 


\title{
EXTENSIONS OF PRO-AFFINE ALGEBRAIC GROUPS
}

\section{Brian Peterson}

\begin{abstract}
Our objective is to make a series of reductions for the problem of computing Ext in the category of pro-affine algebraic groups over an algebraically closed field of characteristic zero, exploiting the notions of unipotence, reductiveness, and group coverings. After examining some of the properties of Ext in a more general categorical setting, due to G. Hochschild, we discuss the multiplicative character theory for our groups and obtain several consequences of simple connectedness before proceeding to the main objective.
\end{abstract}

1. Preliminaries. Let $G$ be a group, $F$ a field, and $g: G \rightarrow F$ an $F$-valued function on $G$. For each $x$ in $G$, we define the left and right translates of $g$ by $x$ by $(x \cdot g)(y)=g(y x)$ and $(g \cdot x)(y)=$ $g(x y)$ for all $y$ in $G$, respectively. For all $x$ and $y$ in $G$, we have $(x \cdot g) \cdot y=x \cdot(g \cdot y)$ so we denote this simply by $x \cdot g \cdot y$. We call $g$ a representative $F$-valued function if the functions $x \cdot g \cdot y$ with $x$ and $y$ ranging over $G$ lie in a finite dimensional space of functions. This condition may easily be shown to be equivalent to the assertion that the functions $x \cdot g$ all lie in a finite dimensional space of functions, or that the functions $g \cdot x$ do. The set $\mathscr{R}_{F}(G)$ of all representative $F$-valued functions on $G$ has the structure of a Hopf algebra over $F$. The $F$-algebra structure of $\mathscr{R}_{F}(G)$ is the usual one. The comultiplication $\gamma: \mathscr{R}_{F}(G) \rightarrow \mathscr{R}_{F}(G) \otimes \mathscr{R}_{F}(G)$ sends any $g$ as above to the unique element $\sum f_{i} \otimes g_{i}$ of $\mathscr{R}_{F}(G) \otimes \mathscr{R}_{F}(G)$ for which $\sum f_{i}(x) g_{i}(y)=g(x y)$ for all $x$ and $y$ in $G$. The antipode $\eta: \mathscr{R}_{F}(G) \rightarrow \mathscr{R}_{F}(G)$ sends $g$ to the function whose value at each $x$ in $G$ is $g\left(x^{-1}\right)$, and the counit $c: \mathscr{R}_{F}(G) \rightarrow F$ is evaluation at the identity element of $G$.

The pair $(G, A)$ is called a pro-affine algebraic group over $F$ if $A$ is a Hopf subalgebra of $\mathscr{R}_{F}(G)$ which separates the points of $G$ and has the property that every $F$-algebra homomorphism $A \rightarrow F$ is the evaluation at some element of $G$. If $A$ is finitely generated we call $(G, A)$ an affine algebraic group over $F$.

More generally, if $X$ is a set and $A$ is an $F$-algebra of $F$-valued functions on $X$, we call the pair $(X, A)$ a pro-affine algebraic variety over $F$ if $A$ separates the points of $X$ and every $F$-algebra homomorphism $A \rightarrow F$ is the evaluation at some element of $X$. If $A$ is finitely generated, we call $(X, A)$ an affine algebraic variety over $F$. This is one equivalent form of the usual notion.

If $(X, A)$ and $(Y, B)$ are pro-affine algebraic varieties over $F$, 
a function $\phi: X \rightarrow Y$ is called a polynomial map if, for each $f$ in $B$, the function $f \circ \phi$ belongs to $A$, in which case the map sending such $f$ to $f \circ \phi$ is an $F$-algebra homomorphism $B \rightarrow A$ which we will denote by $\phi^{\prime}$. If $(G, A)$ and $(H, B)$ are pro-affine algebraic groups over $F$ and $\phi: G \rightarrow H$ is both a polynomial map and a group homomorphism, we call $\phi$ a rational homomorphism. In this case, $\phi^{\prime}$ is a Hopf algebra homomorphism.

We assume from now on that $F$ is algebraically closed, though some of what we say is true more generally.

Since $F$ is algebraically closed, the functor sending $(X, A)$ to $A$ and $\phi$ to $\phi^{\prime}$ is easily seen to be a contravariant categorical equivalence from the category of pro-affine algebraic varieties over $F$ to the category of commutative $F$-algebras which are reduced, i.e., contain no nonzero nilpotent elements. An inverse functor is the one sending the commutative reduced $F$-algebra $A$ to the pair $(\mathscr{G}(A), A)$ where $\mathscr{G}(A)$ is the set of all $F$-algebra homomorphisms $A \rightarrow F$ and elements of $A$ are viewed as functions on $\mathscr{G}(A)$ by evaluation. If $A$ is in fact a Hopf algebra, $\mathscr{G}(A)$ has a natural group structure and the functors defined just as the above are easily seen to establish a contravariant categorical equivalence between the category of pro-affine algebraic groups over $F$ and that of commutative reduced Hopf $F$-algebras.

We will use the notations $(X, A),(X, P(X))$, and $(\mathscr{G}(A), A)$ interchangably for pro-affine algebraic groups and varieties.

We give to pro-affine algebraic groups and varieties $(X, A)$ the usual Zariski topology, so that a subset $Z$ of $X$ is closed (or algebraic) if and only if $Z$ is the full annihilator in $X$ of the annihilator $J$ in $A$ of $Z$. The closure of a subset of $X$ will be called its algebraic hull (in $X$ ). If $Z$ is algebraic, then $(Z, A / J)$ is a pro-affine algebraic variety in the natural way.

If, in the above situation, $(X, A)$ is a pro-affine algebraic group and $Z$ a closed subgroup, then $J$ is a Hopf ideal, $A / J$ a Hopf algebra, and $(Z, A / J)$ a pro-affine algebraic group. If, in addition, $Z$ is normal in $X$, then $\left(X / Z, A^{Z}\right)$ is a pro-affine algebraic group, where $A^{Z}$ denotes the $Z$-fixed part of $A$ under left (equivalently right) translations. The canonical map $\pi: X \rightarrow X / Z$ is a rational homomorphism with $\pi^{\prime}$ the natural inclusion $A^{Z} \rightarrow A$. It is easily seen that if $\rho: X \rightarrow Y$ is a polynomial map to a pro-affine algebraic variety $Y$ which is constant on cosets $\bmod Z$, the induced map $\rho^{Z}: X / Z \rightarrow Y$ with $\rho^{Z} \circ \pi=\rho$ is a polynomial map, and if $\rho$ is a rational homomorphism to a pro-affine algebraic group $Y$, then $\rho^{Z}$ is a rational homomorphism.

If $(X, A)$ and $(Y, B)$ are pro-affine algebraic varieties (or groups) over $F$, the map sending $(\phi, \psi)$ to $\phi \otimes \psi$ gives a bijection $\mathscr{G}(A) \times$ 
$\mathscr{G}(B) \rightarrow \mathscr{G}(A \otimes B)$, and the above contravariant categorical equivalences make it clear that $(X \times Y, A \otimes B)$ is a product in the category of pro-affine algebraic varieties (or groups) over $F$, as tensor products are coproducts in any reasonable category of $F$ algebras.

If $(G, A)$ is a pro-affine algebraic group over $F$ and $H$ a subgroup of $G, H$ is called unipotent if the representation (by left translations) of $H$ on any finite dimensional left $H$-stable subspace of $A$ is unipotent. $H$ is called reductive if its representation on $A$ is semisimple. If $H$ is algebraic and unipotent, it follows that all of its rational representations are locally unipotent, and if $H$ is algebraic and reductive it follows that all of its rational representations are semisimple (cf. [7, Proposition 2.3]).

In [6] we see that there is a normal unipotent (algebraic) subgroup of $G$, which we will denote $G_{u}$, which contains all normal unipotent subgroups of $G$. Any reductive subgroup of $G$ intersects $G_{u}$ trivially. It is shown that if $F$ is of characteristic zero (and algebraically closed), there is a reductive algebraic subgroup $K$ of $G$ such that $G$ is the semidirect product $G_{u} K$, and any reductive subgroup $L$ of $G$ has some conjugate $x L x^{-1}$ contained in $K$ with $x$ in the algebraic hull of the commutator subgroup $\left[G_{u}, G\right]$. So if $G$ is abelian, there is a unique maximal (algebraic) reductive subgroup $G_{r}$ of $G$ and $G=G_{u} \times G_{r}$.

A tool which is used in the proofs of most of these results is Proposition 2.7 of [7], which we record here.

Projective Limit Theorem. Let $\left(V_{\alpha}, \rho_{\alpha}^{\beta}\right)$ be an inverse system of compact $T_{1}$ topological spaces with continuous closed maps $\rho_{\alpha}^{\beta}: V_{\beta} \rightarrow V_{\alpha}$ when $\alpha \leqq \beta$. This means, of course, that for any $\alpha$ and $\beta$, there is a $\gamma$ with $\alpha \leqq \gamma$ and $\beta \leqq \gamma$, that each $\rho_{\alpha}^{\alpha}$ is the identity on $V_{\alpha}$, and that $\rho_{\alpha}^{\beta} \circ \rho_{\beta}^{\gamma}=\rho_{\alpha}^{\gamma}$ when $\alpha \leqq \beta \leqq \gamma$. Then the inverse (projective) limit $V$ of the system is nonempty. If, for some $\alpha$, all the maps $\rho_{\alpha}^{\beta}$ with $\beta \geqq \alpha$ are surjective, so is the cononical $\operatorname{map} \rho_{\alpha}: V \rightarrow V_{\alpha}$.

If $(G, A)$ is a pro-affine algebraic group over $F$, every finitely generated subalgebra of $A$ is contained in a finitely generated Hopf subalgebra, so $A$ is the union of these latter. If $S$ ranges over any increasingly filtered family of these whose union is $A$, we have a natural identification $\mathscr{G}(A)=\underset{\leftarrow}{\lim } \mathscr{G}(S)$. Each $(\mathscr{G}(S), S)$ is an affine algebraic group over $F$, hence the name pro-affine algebraic group. A similar (simpler) discussion applies to pro-affine algebraic varieties. Since we assume $F$ is algebraically closed, the projective limit theo- 
rem applies to inverse systems in the category of affine algebraic groups over $F$, via the coset topology (cf. [7]).

A pro-affine algebraic group $(G, A)$ over $F$ is called connected if $A$ is an integral domain. Proposition 2.1 of [6] shows that in general $G$ contains a unique normal connected algebraic subgroup $G_{1}$ such that $G / G_{1}$ is pro-finite, i.e., such that the restriction image of $G / G_{1}$ to any finitely generated Hopf subalgebra of $A$ is finite. $G_{1}$ is called the identity component of $G$, and its annihilator in $A$ consists of all elements $a$ of $A$ for which there is an $a^{\prime}$ in $A$ with $a^{\prime}(1) \neq 0$ and $a a^{\prime}=0$.

2. Group coverings. Let $(G, A)$ and $(H, B)$ be connected proaffine algebraic groups over $F$. A surjective rational homomorphism $\pi: H \rightarrow G$ is called a covering if its kernel $P$ is pro-finite, i.e., $P_{1}=(1)$, and the induced rational homomorphism $H / P \rightarrow G$ is a rational isomorphism. If $F$ has characteristic 0 , any bijective rational homomorphism is a rational isomorphism, so the latter condition is automatically satisfied. It follows from the fact that $P$ is a totally disconnected normal algebraic subgroup of the connected group $H$ that $P$ is central in $H$. Because $\pi$ is surjective, the induced Hopf algebra homomorphism $\pi^{\prime}: A \rightarrow B$ is injective.

In the case where $F$ has characteristic 0 , necessary and sufficient conditions are given in [4] that, for a commutative integral domain Hopf algebra $B$ over $F$ containing the Hopf subalgebra $A$, the restriction map $\mathscr{G}(B) \rightarrow \mathscr{G}(A)$ is a group covering, namely that $B$ is what is called a profinite extension of $A$. We refer the reader to [4] for the details, including the definition of the space $D_{s}(T)$ of Kahler differentials of an $F$-algebra $T$ relative to an $F$-subalgebra $S$, which we will take for granted in the proofs of Lemma 4.3 and Theorem 4.4 below.

We remark that, again if $F$ has characteristic 0 , the proof of Theorem 4.2 of [4] shows that if the integral domain $F$-algebra $B$ is a profinite extension of the Hopf $F$-algebra $A$, then for any $F$ algebra homomorphism $c^{\prime}: B \rightarrow F$ extending the counit $c: A \rightarrow F$ of $A, B$ has a unique structure of Hopf $F$-algebra having $c^{\prime}$ as counit, i.e., the identity element of $\mathscr{G}(B)$, which extends the Hopf algebra structure of $A$.

A connected pro-affine algebraic group $G$ over the algebraically closed field $F$ is called simply connected if every covering of $G$ is an isomorphism. If $G$ and $H$ are connected pro-affine algebraic groups over $F$, a covering $\pi: H \rightarrow G$ is called a universal covering if, for every covering $\tau: T \rightarrow G$, there is a unique covering $\pi^{\prime}: H \rightarrow T$ such that $\tau \circ \pi^{\prime}=\pi$. If $\pi: H \rightarrow G$ is a universal covering, it is easy to see that $H$ must be simply connected. Theorem 5.1 of [4] shows 
that every connected pro-affine algebraic group over $F$ has a universal covering, which is unique up to unique isomorphism. Theorem 5.2 of [4] shows that if $G$ is a connected pro-affine algebraic group over $F$ and $K$ a connected normal algebraic subgroup of $G$, then if both $K$ and $G / K$ are simply connected so is $G$, and if $G$ is simply connected so is $G / K$, and so also is $K$ if $F$ is of characteristic 0 (as well as algebraically closed).

To see that a unipotent pro-affine algebraic group over an algebraically closed field of characteristic 0 is simply connected, we suppose that $G$ is one and $\pi: H \rightarrow G$ a covering. We have $H=H_{u} \cdot H_{r}$ where $H_{r}$ is a maximal reductive subgroup of $H$. Since $\pi\left(H_{r}\right)$ is reductive and $G$ unipotent, $\pi\left(H_{r}\right)=(1)$. But $H$, hence $H_{r}$, is connected while the kernel of $\pi$ is profinite. Hence $H_{r}=(1)$, so $H$ is unipotent. Thus the kernel of $\pi$, being both reductive and unipotent, is trivial.

It is well-known (c.f. [1]) that if $G$ is a connected reductive affine algebraic group over an algebraically closed field $F$ of characteristic zero and $C_{1}(G)$ denotes the identity component of the center of $G$ and $G^{\prime}=[G, G]$, then both $C_{1}(G)$ and $G^{\prime}$ are algebraic subgroups of $G$ and the multiplication map $C_{1}(G) \times G^{\prime} \rightarrow G$ is a covering, i.e., a surjective rational homomorphism with finite kernel. If $(G, A)$ is a connected reductive pro-affine algebraic group over such a field $F$, let $S$ range over the set of all finitely generated Hopf subalgebras of $A$. Let $G^{\prime}$ denote the inverse limit of all the $\left(G_{S}\right)^{\prime \prime}$,s, so that $G^{\prime}$ is the algebraic hull of the commutator subgroup of $G$. We have that the identity component $C_{1}(G)$ of the center of $G$ is equal to the inverse limit of the $C_{1}\left(G_{S}\right)$ 's. It follows that the multiplication map $C_{1}(G) \times G^{\prime} \rightarrow G$ is a covering whose kernel is the inverse limit of the kernels of the maps $C_{1}\left(G_{S}\right) \times\left(G_{S}\right)^{\prime} \rightarrow G_{S}$. We record this as a lemma.

Lemma 1.1. Let $G$ be a connected reductive pro-affine algebraic group over an algebraically closed field $F$ of characteristic $0 . \quad$ Let $C_{1}(G)$ denote the identity component of the center of $G$ and $G^{\prime}$ the algebraic hull of the commutator subgroup of $G$. Then the multiplication map $C_{1}(G) \times G^{\prime} \rightarrow G$ is a covering. If $G$ is simply connected this map is rational isomorphism.

3. Automorphism groups. Let $F$ be an algebraically closed field and $(G, A)$ a pro-affine algebraic group over $F$. Let Aut $(G)$ denote the group of all rational automorphisms of $G$. For $\phi$ in Aut $(G)$ and $a$ in $A$, we define the induced right translate $a \cdot \phi$ of $a$ by $\dot{\phi}$ by $a \cdot \dot{\phi}=a \circ \dot{\phi}$.

Call a subgroup $P$ of Aut $(G)$ an algebraic subgroup of Aut $(G)$ 
if $P$ has a structure $(P, B)$ of pro-affine algebraic group over $F$ such that the map $P \times G \rightarrow G$ sending each $(\phi, x)$ to $\phi(x)$ is a polynomial map. Theorem 2.1 of [2] says that a subgroup $P$ of $\operatorname{Aut}(G)$ for which $A$ becomes a locally finite $P$-module by right translations is contained in a unique minimal algebraic subgroup of Aut $(G)$. If $P$ is itself and algebraic subgroup of Aut $(G)$, the same theorem shows that there is a smallest Hopf subalgebra $A(P)$ of $\mathscr{R}_{F}(P)$ making $(P, A(P))$ an algebraic subgroup of Aut $(G)$, and that $A(P)$ is the smallest Hopf subalgebra of $\mathscr{R}_{F}(P)$ containing the functions $\lambda / a$ with $\lambda$ in $\operatorname{Hom}_{F}(A, F)$ and $a$ in $A$, where $\lambda / a(\phi)=\lambda(\alpha \circ \phi)$ for $\phi$ in $P$. If the characteristic of $F$ is 0 and the structure $(P, B)$ makes $P$ an algebraic subgroup of $\operatorname{Aut}(G)$, then necessarilly $B=A(P)$.

Proposition 2.2 of [2] says that if $(P, A(P))$ is an algebraic subgroup of $\operatorname{Aut}(G)$ as above, and if $H$ is an algebraic $P$-stable subgroup of $G$, then the canonical restriction map $P \rightarrow \operatorname{Aut}(H)$ is a rational homomorphism onto an algebraic subgroup $\left(P_{H}, A\left(P_{H}\right)\right)$ of Aut $(H)$. If $H$ is normal in $G$, the canonical map $P \rightarrow \operatorname{Aut}(G / H)$ is a rational homomorphism onto an algebraic subgroup $\left(P_{G / H}, A\left(P_{G / H}\right)\right)$ of $\operatorname{Aut}(G / H)$.

Proposition 2.3 of [2] says that if $P$ is an algebraic subgroup of Aut $(G)$ as above, then every finitely generated Hopf subalgebra of $A$ is contained in a finitely generated $P$-stable Hopf subalgebra of $A$. If $B$ is a finitely generated $P$-stable Hopf subalgebra of $A$, the canonical map $P \rightarrow \operatorname{Aut}\left(G_{B}\right)$ is a rational homomorphism onto an affine algebraic subgroup of Aut $\left(G_{B}\right)$.

It is implicit in [2] that if $\phi: G \rightarrow \operatorname{Aut}(H)$ is a homomorphism, where $G$ and $H$ are pro-affine algebraic groups over $F$, then $\phi$ is a rational homomorphism onto an algebraic subgroup of $\operatorname{Aut}(H)$ if and only if the map sending $(g, h)$ to $\phi(g)\{h\}$ is a polynomial map $G \times H \rightarrow H$.

4. Two remarks on pro-affine varieties. For each pro-affine algebraic variety $(X, A)$ over $F$, we have the diagonal map $d: X \rightarrow$ $X \times X$ where $d(x)=(x, x)$ for $x$ in $X$. The image $d(X)$ of $d$ is easily seen to be an algebraic subset of $X \times X$ whose annihilator in $A \otimes A$ is the ideal generated by all elements $a \otimes 1-1 \otimes a$ with $a$ in $A$. The map $X \rightarrow d(X)$ induced by $d$ is a polynomial isomorphism whose inverse is the restriction to $d(X)$ of either natural projection $X \times X \rightarrow X$. So the diagonal map is a closed morphism onto a closed subset of $X \times X$, i.e., $X$ is separated in the usual algebraic geometric sence. It follows that for any two polynomial maps $f_{1}, f_{2}: X \rightarrow Y$, the set of all elements $x$ of $X$ with $f_{1}(x)=f_{2}(x)$ is an algebraic subset of $X$.

The following lemma will be useful in $\S 2$. Suppose $(X, A)$ is a 
pro-affine algebraic variety over $F$ and that $Z$ is an algebraic subset of $X$ with annihilator $I$ in $A$. Let $X I X$ denote the disjoint union of two copies of $X$ and let $X \amalg_{z} X$ denote the set of equivalence classes in $X \amalg X$ where two elements are equivalent if and only if they represent the same element of $Z$ in the respective copies of $X$. Let $f_{1}$ and $f_{2}$ denote the two maps $X \rightarrow X \Pi_{z} X$ induced by the two inclusions $X \rightarrow X \amalg X$ of $X$ into the first, respectively second, copy of $X$.

LEMMA 1.2. $X \coprod_{z} X$ possesses a structure of pro-affine algebraic variety over $F$ such that $f_{1}$ and $f_{2}$ are polynomial map and $\left(X \amalg_{Z} X, f_{1}, f_{2}\right)$ has the universal property that for any triple $\left(Y, g_{1}, g_{2}\right)$ where $Y$ is a pro-affine algebraic variety over $F$ and $g_{1}$ and $g_{2}$ are two polynomial maps $X \rightarrow Y$ whose restrictions to $Z$ coincide, there is a unique polynomial map $k: X \amalg_{z} X \rightarrow Y$ such that $k \circ f_{1}=g_{1}$ and $k \circ f_{2}=g_{2}$. Also, clearly, $Z$ coincides with the set of elements $x$ of $X$ for which $f_{1}(x)=f_{2}(x)$.

Proof. Consider the $F$-algebra $A \oplus A$. Let $A \bigoplus_{I} A$ denote the $F$-subalgebra of $A \oplus A$ consisting of all elements $\left(a_{1}, a_{2}\right)$ with $a_{1}+$ $I=a_{2}+I$. Both $A \oplus A$ and $A \bigoplus_{I} A$ are clearly reduced. Two $F$ algebra homomorphisms $A \oplus A \rightarrow F$ are equal if and only if their kernels coincide, and the same is true for $A \oplus_{I} A$. The kernel of any $F$-algebra homomorphism $A \oplus A \rightarrow F$ contains either $(1,0)$ or $(0,1)$ since it contains their product. So if $\pi_{1}$ and $\pi_{2}$ are the canonical projections $A \oplus A \rightarrow A$, then any $F$-algebra homomorphism $A \oplus A \rightarrow F$ factors through either $\pi_{1}$ or $\pi_{2}$. We therefore have a natural identification of $X I X$ with the set of all $F$-algebra homomorphisms $A \oplus A \rightarrow F$.

Because $F$ is algebraically closed, any $F$-algebra homomorphism $A \bigoplus_{I} A \rightarrow F$ extends to one $A \oplus A \rightarrow F$, but not always uniquely. If $m$ and $m^{\prime}$ are the kernels of two distinct homomorphisms $A \rightarrow F$, then neither $(m \oplus A) \cap\left(A \bigoplus_{I} A\right)$ nor $(A \oplus m) \cap\left(A \oplus_{I} A\right)$ is equal to either $\left(m^{\prime} \oplus A\right) \cap\left(A \bigoplus_{I} A\right)$ or $\left(A \oplus m^{\prime}\right) \cap\left(A \bigoplus_{I} A\right)$ because, for example, if $a$ belongs to $m$ but not $m^{\prime}$, then $(a, a)$ is in both of the former and neither of the latter. Now if $(m \oplus A) \cap\left(A \bigoplus_{I} A\right)=$ $(A \oplus m) \cap\left(A \bigoplus_{I} A\right)$, then for every $a$ in $I$ we have $(a, 0) \in(A \oplus m) \cap$ ' $\left(A \bigoplus_{I} A\right)$ so also $(a, 0) \in(m \oplus A) \cap\left(A \bigoplus_{I} A\right)$ and $a \in m$. Thus $I \leqq m$. Clearly if $I \subseteq m$ then $(m \oplus A) \cap\left(A \bigoplus_{I} A\right)=(A \oplus m) \cap\left(A \bigoplus_{I} A\right)$. Thus an $F$-algebra homomorphism $A \bigoplus_{I} A \rightarrow F$ has either one or two extensions to $A \oplus A$ according as its kernel does not or does contain $I \oplus I$. If its kernel does contain $I \oplus I$, it is of the form $m \bigoplus_{I} m$ and the kernels of the two extensions to $A \oplus A$ are $m \oplus A$ and $A \oplus m$. This shows that via the identification of $X I I X$ 
with $\operatorname{Hom}_{\text {alg }}(A \oplus A, F)$, the restriction map $\operatorname{Hom}_{\text {alg }}(A \oplus A, F) \rightarrow$ $\mathrm{Hom}_{\mathrm{alg}}\left(A \bigoplus_{I} A, F\right)$ identifies $X \amalg_{Z} X$ with $\operatorname{Hom}_{\mathrm{alg}}\left(A \bigoplus_{I} A, F\right)$. Via this identification, the maps $f_{1}$ and $f_{2}$ correspond to the restrictions to $A \bigoplus_{I} A$ of $\pi_{1}$ and $\pi_{2}$, so both $f_{1}$ and $f_{2}$ are polynomial maps.

The universal property of $\left(X \amalg_{z} X, f_{1}, f_{2}\right)$ is now easy to deduce. Since we will not use it, we omit the argument. The last assertion is clear.

5. Functorial properties of Ext. The results of this section are valid in the setting described in $\S 2$ of [3]. We remind the reader that $\mathscr{S}$ is a category of spaces (sets with possibly some additional structure), $\mathscr{S}_{g}$ the category of groups in $\mathscr{S}$ (i.e., objects of $\mathscr{S}$ with group structures suth that multiplication and inversion are $\mathscr{S}$ morphisms), and $\mathscr{G}$ is a full subcategory of $\mathscr{S}_{g}$. Several assumptions are made on $\mathscr{S}$ and $\mathscr{G}$, for example, that both admit (finite) products and that $\mathscr{G}$ admits semidirect products (cf. [3]). The above provides all that is needed to verify that all of the assumptions are satisfied when $\mathscr{S}$ is the category of pro-affine algebraic varieties over an algebraically closed field $F$ of characteristic zero and $\mathscr{G}$ is either $\mathscr{S}_{g}$ or the category of affine algebraic groups over $F$.

We recall that for objects $G$ of $\mathscr{G}$ and $A$ of $\mathscr{S}, A$ is called a $G$-space if there is given a homomorphism $\gamma: G \rightarrow \operatorname{Aut}_{\mathscr{S}}(A)$ such that the induced map $G \times A \rightarrow A$ is an $\mathscr{S}$-morphism. If $A$ belongs to $\mathscr{G}$ and $\gamma(G) \subset$ Aut, $_{,}(A), A$ is called a $G$-group. We recall also that it is assumed that every bijective $\mathscr{G}$-morphism is a $\mathscr{G}$-isomorphism.

A subset $B$ of an object $A$ of $\mathscr{S}$ is called closed in $A$ if for every $a$ in $A$ and not in $B$, there are $\mathscr{S}$-morphisms $f$ and $g$ from $A$ to some object $C$ of $\mathscr{S}$ with $f(b)=g(b)$ for all $b$ in $B$ but $f(a) \neq$ $g(a)$. The discussion of $\S 5$ shows that in the category of pro-affine algebraic groups over $F$ as above, this notion of closure is equivalent with Zariski closure. In general, enough assumptions are made on $\mathscr{S}$ that this gives its objects $T_{1}$ topologies such that all $\mathscr{S}$-morphisms are continuous.

Although it is not necessary, we avoid technical difficulties by assuming that if $K$ is a closed normal subgroup of an object $G$ of $\mathscr{G}$, then for any $\mathscr{S}$-morphism $G \rightarrow A$ ( $A$ an object of $\mathscr{S}$ ) which is constant on cosets $\bmod K$, the induced map $G / K \rightarrow A$ is an $\mathscr{S}$-morphism. It follows that if $K$ acts trivially on a $\mathscr{G}$-space $A$, then $A$ is a $G / K$-space. Also, one easily sees that a $G$-stable subspace of a $G$-space is also a $G$-space. We have seen above that these conditions are satisfied in the categories of pro-affine algebraic groups over a field $F$ as above. 
Group extensions. If $A$ and $H$ are objects of $G$ an extension of $A$ by $H$ consists of a surjective $\mathscr{G}$-morphism $\sigma: E \rightarrow H$ and a $\mathscr{G}$ isomorphism $\alpha: A \rightarrow E$ of $A$ onto the kernel of $\sigma$. It follows that the induced bijective $\mathscr{G}$-morphism $E / \alpha(A) \rightarrow H$ is a $\mathscr{G}$-isomorphism (which would otherwise have been part of our definition). Such an extension will be denoted by $[A, E, H]_{\alpha, \sigma}$, or $[A, E, H]_{\sigma}$ when we wish to suppress $\alpha$ in the notation. We will generally regard $\alpha$ as an identification of $A$ with $\alpha(A)$ making the abbreviated notation natural, as well as more controllable. Two extensions $\left[A, E_{1}, H\right]_{\sigma_{1}}$ and $\left[A, E_{2}, H\right]_{\sigma_{2}}$ will be called equivalent if there is a $\mathscr{G}$-morphism $\lambda: E_{1} \rightarrow E_{2}$ such that $\lambda(a)=a$ for each $a$ in $A$ and $\sigma_{2} \circ \lambda=\sigma_{1}$, which makes $\lambda$ a $\mathscr{G}$-isomorphism.

For an object $A$ of $\mathscr{G}$, we will write $\operatorname{Aut}(A)$ for $\operatorname{Aut},(A)$ and Inn $(A)$ for the normal subgroup of $\operatorname{Aut}(A)$ consisting of all inner automorphisms of $A$. We shall write $O(A)$ for $\operatorname{Aut}(A) / \operatorname{Inn}(A)$.

Let $[A, E, H]_{\sigma}$ be a group extension in $\mathscr{G}$. It follows that the map $A \rightarrow A$ sending $a$ to $e a e^{-1}$ is a $\mathscr{G}$-automorphism for each $e \in E$. So we have a homomorphism $E \rightarrow \operatorname{Aut}(A)$ which is easily seen to make $A$ into an $E$-group. The kernel of the induced homomorphism $E \rightarrow O(A)$ contains $A$, so this induces a homomorphism $\eta: H \rightarrow O(A)$. We say that the extension $[A, E, H]_{\sigma}$ induces $\eta$. One easily sees that equivalent extensions of $A$ by $H$ induce the same homomorphism $\eta$, so we may speak of the homomorphism $\eta$ induced by an equivalence class of extensions of $A$ by $H$. We denote by $\operatorname{Ext}(H, A, \eta)$ the set of all equivalence classes of extensions of $A$ by $H$ inducing $\eta: H \rightarrow O(A)$, which may be empty for a particular homomorphism $\eta$.

If $A$ happens to be abelian, then $\operatorname{Inn}(A)$ is trivial, so $\eta: H \rightarrow$ Aut $(A)$. More generally, let $[A, E, H]_{\sigma}$ be a group extension in $\mathscr{G}$ inducing $\eta: H \rightarrow O(A)$. Let $C$ denote the center of $A$. One easily sees that $C$ is a closed normal subgroup of $A$. Clearly $C$ is stable under every element of Aut $(A)$ and, in fact, restriction gives a homomorphism Aut $(A) \rightarrow \operatorname{Aut}(C)$ which is trivial on Inn $(A)$, hence induces a homomorphism $O(A) \rightarrow$ Aut $(C)$. The induced $E$-group structure on $C$ is the composite $E \rightarrow \operatorname{Aut}(A) \rightarrow \operatorname{Aut}(C)$ and this is trivial on $A$, so induces an $H$-group structure $\eta_{0}: H \rightarrow$ Aut $(C)$. This is easily seen to coincide with the composite $H \stackrel{\eta}{\rightarrow} O(A) \rightarrow \operatorname{Aut}(C)$.

Next we describe the Baer composite, which gives to $\operatorname{Ext}\left(H, C, \eta_{0}\right)$ the structure of abelian group and also gives an action of this group on the set $\operatorname{Ext}(H, A, \eta)$. We will then prove the analogue of the familiar result in the category of abstract groups that this action is faithful and (simply) transitive.

Suppose $[A, E, H]_{\sigma}$ and $[B, F, H]_{\tau}$ are two extensions in $\mathscr{G}$, inducing $\eta: H \rightarrow O(A)$ and $\mu: H \rightarrow O(B)$, respectively. Let $C(A)$ and $C(B)$ denote the respective centers. Suppose that $C$ is a object of 
$C^{3}$ which is a closed subgroup of both $C(A)$ and $C(B)$ and that the induced actions $\eta_{0}: H \rightarrow$ Aut $(C(A))$ and $\mu_{0}: H \rightarrow \operatorname{Aut}(C(B))$ both stabilize $C$ and induce the same action of $H$ on $C$ making $C$ an $H$-group.

Let $C^{0}$ denote the set of all elements $\left(c, c^{-1}\right)$ of $A \times B$ with $c \in C$. Then $C^{0}$ is a central subgroup of $A \times B$ which is easily seen to be closed. Write $D$ for $(A \times B) / C^{0}$. Because we assume that $\eta$ and $\mu$ induce the same action on $C$, they canonically induce a homomorphism $\nu: H \rightarrow O(D)$. Namely, if $\phi$ and $\psi$ represent $\eta(h)$ and $\mu(h)$ in $\operatorname{Aut}(A)$ and $\operatorname{Aut}(B)$, respectively, the element $\dot{\phi} \times \psi$ of $\operatorname{Aut}(A \times B)$ stabilizes $C^{0}$ (and so does its inverse) and so induces an element of Aut $(D)$. A routine check shows that the class $\nu(h)$ in $O(D)$ of this element depends only on $\eta(h)$ and $\mu(h)$, and that this indeed defines a homomorphism $\nu: H \rightarrow O(D)$. The map $C \rightarrow D$ sending $c$ to $(c, 1) C^{0}=$ $(1, c) C^{0}$ is an injective $\mathscr{G}$-morphism onto a closed central subgroup of $D$, so identifies $C$ with a closed central subgroup of $D$ on which $\nu$ is easily seen to induce the same action as $\eta$ and $\mu$.

Now denote by $(E \times F)_{o, \tau}$ the set of all elements $(e, f)$ of $E \times F$ for which $\sigma(e)=\tau(f)$. Then $(E \times F)_{\sigma, \tau}$ is easily seen to be a closed subgroup of $E \times F$. The homomorphism $\pi^{\prime}:(E \times F)_{\sigma, \tau} \rightarrow H$ sending $(e, f)$ to $\sigma(e)=\tau(f)$ is easily seen to be a surjective $\mathscr{G}$-morphism. The kernel of $\pi^{\prime}$ coincides with $A \times B$. Our assumption that $\eta$ and $\mu$ induce the same action on $C$ implies that $C^{0}$ is a (closed) normal subgroup of $(E \times F)_{\sigma, \tau}$. Let $G=(E \times F)_{\sigma, \tau} / C^{0}$ and let $\pi: G \rightarrow H$ be the $\mathscr{G}$-morphism induced by $\pi^{\prime}$. The kernel of $\pi$ coincides with $D=(A \times B) / C^{0}$ and we have an extension $[D, G, H]_{\pi}$ in $\mathscr{G}$ which one easily sees to induce $\nu$. We call $[D, G, H]_{\pi}$ the Baer composite of $[A, E, H]_{\sigma}$ and $[B, F, H]_{\tau}$, and we denote $[D, G, H]_{\pi}$ by $[A, E, H]_{\sigma} \boxplus$ $[B, F, H]_{\tau}$.

Next, a routine verification shows that the class of $[D, G, H]_{\pi}$ in $\operatorname{Ext}(H, D, \nu)$ depends only on the classes of $[A, E, H]_{\sigma}$ and $[B, F, H]_{\tau}$ in $\operatorname{Ext}(H, A, \eta)$ and $\operatorname{Ext}(H, B, \mu)$, respectively, so that the Baer composite yields a map $\operatorname{Ext}(H, A, \eta) \times \operatorname{Ext}(H, B, \mu) \rightarrow \operatorname{Ext}(H, D, \nu)$. Finally, one verifies that this composition is associative, i.e., if $\left[A_{i}, E_{i}, H\right]_{\sigma_{i}}$ represent elements of $\operatorname{Ext}\left(H, A_{i}, \eta_{i}\right)$ for $i=1,2,3$ where $C$ is closed central subgroup of each $A_{i}$ and each $\eta_{i}$ induces the same action of $H$ on $C$, then the class of

$$
\left[A_{1}, E_{1}, H\right]_{\sigma_{1}} \boxplus\left[A_{2}, E_{2}, H\right]_{\sigma_{2}} \boxplus\left[A_{3}, E_{3}, H\right]_{\sigma_{3}}
$$

does not depend on how one inserts parentheses. The verification that the recipe

$$
\left(\left(e_{1}, e_{2}\right) C^{0}, e_{3}\right) C^{0} \longrightarrow\left(e_{1},\left(e_{2}, e_{3}\right) C^{0}\right) C^{0}
$$

does produce the required equivalence is a straightforward series of 
applications of the assumptions on $\mathscr{G}$.

We specialize first to the case where $A$ is abelian, so $\eta: H \rightarrow$ Aut $(A)$. Taking $C=A$, we have a $\mathscr{G}$-isomorphism $(A \times A) / A^{0} \rightarrow A$ induced by the multiplication in $A$. Viewing this isomorphism as an identification, the Baer composite gives a map $\operatorname{Ext}(H, A, \eta) \times$ $\operatorname{Ext}(H, A, \eta) \rightarrow \operatorname{Ext}(H, A, \eta)$. We have just remarked that this operation is associative, and it is easily seen to be commutative. To see that the class of the semidirect product $(A \cdot H)_{\eta}$ is an identity element, one shows that if $[A, E, H]_{\sigma}$ represents an element of $\operatorname{Ext}(H, A, \eta)$, then the recipe $((a, h), e) A^{0} \rightarrow a e$ gives an equivalence $\left[A,(A \cdot H)_{\eta}, H\right] \boxplus[A, E, H]_{\sigma} \rightarrow[A, E, H]_{\sigma}$. Next, if $[A, E, H]_{\sigma}$ represents an element of $\operatorname{Ext}(H, A, \eta)$, a representative for the inverse of this element is given as follows: Let $\alpha: A \rightarrow A$ be the map sending each $a$ in $A$ to $a^{-1}$. Since $A$ is abelian this is a $\mathscr{G}$-isomorphism. We see immediately that the extension $[A, E, H]_{\alpha, \sigma}$ induces $\eta$. Writing $[A, F, H]_{\tau}=[A, F, H]_{\sigma} \boxplus[A, E, H]_{\alpha, \sigma}$, we see that $F=(E \times E)_{\sigma, \sigma} / A^{0}$ where $A^{0}$ is the set of all elements $(a, a)$ with $a$ in $A$. The recipe $\left(e_{1}, e_{2}\right) A^{0} \mapsto\left(e_{1} e_{2}^{-1}, \sigma\left(e_{1}\right)\right)$ is easily seen to give an equivalence $[A, F, H]_{\tau} \rightarrow$ $\left[A,(A \cdot H)_{\eta}, H\right]$. Thus the class of $[A, E, H]_{\alpha, o}$ is inverse to the class of $[A, E, H]_{\sigma}$. So the Baer composite makes $\operatorname{Ext}(H, A, \eta)$ into an abelian group when $A$ is abelian.

More generally, let $[A, E, H]_{\sigma}$ be an extension of $A$ by $H$ in $\mathscr{G}$ inducing $\eta: H \rightarrow O(A)$ and let $C$ denote the center of $A$. We view the $\mathscr{G}$-isomorphism $(C \times A) / C^{0} \rightarrow A$ induced by the restriction to $C \times A$ of the multiplication in $A$ as an identification. Its inverse is the map sending each $a$ in $A$ to $(1, a) C^{0}$. The Baer composite then gives a function $\operatorname{Ext}\left(H, C, \eta_{0}\right) \times \operatorname{Ext}(H, A, \eta) \rightarrow \operatorname{Ext}(H, A, \eta)$. An equivalence $\left[C,(C \cdot H)_{\eta_{0}}, H\right] \boxplus[A, E, H]_{\sigma} \rightarrow[A, E, H]_{\sigma}$ can be constructed in $\mathscr{G}$ from the recipe $((c, h), e) C^{0} \mapsto c e$. This and the associativity of the Baer composite imply that the above function gives an action of the abelian group $\operatorname{Ext}\left(H, C, \eta_{0}\right)$ on the $\operatorname{set} \operatorname{Ext}(H, A, \eta)$.

Proposition 2.1. If $\operatorname{Ext}(H, A, \eta)$ is nonempty, the above action of $\operatorname{Ext}\left(H, C, \eta_{0}\right)$ on $\operatorname{Ext}(H, A, \eta)$ is faithful and (simply) transitive.

Proof. First we show that if $[C, F, H]_{\tau}$ and $[A, E, H]_{\sigma}$ represent elements of $\operatorname{Ext}\left(H, C, \eta_{0}\right)$ and $\operatorname{Ext}(H, A, \eta)$, respectively, and if $[C, F, H]_{\tau} \boxplus[A, E, H]_{\sigma}$ is equivalent to $[A, E, H]_{o}$, then $[C, F, H]_{\tau}$ is equivalent to $\left[C,(C \cdot H)_{\eta_{0}}, H\right]$. This implies that the action is faithful.

Write $[A, G, H]_{\rho}$ for $[C, F, H]_{\tau} \boxplus[A, E, H]_{\sigma}$ and suppose $\alpha: G \rightarrow E$ is an equivalence isomorphism. The map $\beta:(F \times E)_{\tau, \sigma} \rightarrow E$ given by $\beta(f, e)=e \alpha\left\{(f, e) C^{0}\right\}^{-1}$ is an $\mathscr{S}$-morphism. Now $\sigma \beta(f, e)=$ $\sigma(e) \sigma \alpha\left\{(f, e) C^{0}\right\}^{-1}=\sigma(e) \rho\left\{(f, e) C^{0}\right\}^{-1}=\sigma(e) \sigma(e)^{-1}=1$ for all $(f, e) \in(F \times E)_{\tau, \sigma}$, so $\beta(f, e) \in A$ for all $(f, e) \in(F \times E)_{\tau, \sigma}$. Also; whenever $(f, e) \in(F \times E)_{\tau, \sigma}$ 
and $a \in A$, we have $e^{-1} a e=\alpha\left\{\left(1, e^{-1} a e\right) C^{0}\right\}=\alpha\left\{(f, e)^{-1}(1, a)(f, e) C^{0}\right\}=$ $\alpha\left\{(f, e) C^{0}\right\}^{-1} a \alpha\left\{(f, e) C^{0}\right\}$ which implies that $a^{-1} \beta(f, e) a=\beta(f, e)$. Thus $\beta(f, e) \in C$, and $\beta$ may be viewed as an $\mathscr{S}$-morphism $(F \times E)_{\tau, \sigma} \rightarrow C$. Now we define an $\mathscr{S}$-morphism $\gamma:(F \times E)_{\tau, \sigma} \rightarrow F$ by $\gamma(f, e)=\beta(f, e) f$. If $\left(f_{1}, e_{1}\right)$ and $\left(f_{2}, e_{2}\right)$ are elements of $(F \times E)_{\tau, \sigma}$, then

$$
\begin{aligned}
\gamma\left(f_{1}, e_{1}\right) \gamma\left(f_{2}, e_{2}\right) & =e_{1} \alpha\left\{\left(f_{1}, e_{1}\right) C^{0}\right\}^{-1} f_{1} e_{2} \alpha\left\{\left(f_{2}, e_{2}\right) C^{0}\right\}^{-1} f_{2} \\
& =e_{1} \alpha\left\{\left(f_{1}, e_{1}\right) C^{0}\right\}^{-1} \eta_{0}\left(\tau\left(f_{1}\right)\right)\left\{e_{2} \alpha\left\{\left(f_{2}, e_{2}\right) C^{0}\right\}^{-1}\right\} f_{1} f_{2} \\
& =e_{1} \alpha\left\{\left(f_{1}, e_{1}\right) C^{0}\right\}^{-1} \eta_{0}\left(\rho\left(\left(f_{1}, e_{1}\right) C^{0}\right)\right)\left\{e_{2} \alpha\left\{\left(f_{2}, e_{2}\right) C^{0}\right\}^{-1}\right\} f_{1} f_{2} \\
& =e_{1} e_{2} \alpha\left\{\left(f_{2}, e_{2}\right) C^{0}\right\}^{-1} \alpha\left\{\left(f_{1}, e_{1}\right) C^{0}\right\}^{-1} f_{1} f_{2}=\gamma\left(f_{1} f_{2}, e_{1} e_{2}\right)
\end{aligned}
$$

so $\gamma$ is a $\mathscr{G}$-morphism. If $c \in C$ and $a \in A$, then $\gamma\left(c, a c^{-1}\right)=$ $a c^{-1} \alpha\left\{(1, a) C^{0}\right\}^{-1} C=1$, so the closed normal subgroup $(1, A) C^{0}$ of $(F \times E)_{\tau, \sigma}$ is contained in the kernel of $\gamma$. Hence $\gamma$ induces a $\mathscr{G}$ morphism $\delta: H \rightarrow F$ such that $\delta \rho\left((f, e) C^{0}\right)=\gamma(f, e)$, i.e., $\delta \tau(f)=\gamma(f, e)$. Thus $\tau \delta \tau(f)=\tau(\gamma(f, e))=\tau(f)$. Since $\tau$ is surjective, this shows that $\tau \delta$ is the identity map on $H$. Now the map $F \rightarrow(C \cdot H)_{\eta_{0}}$ given by $f \rightarrow\left(f \delta \tau(f)^{-1}, \tau(f)\right)$ is an $\mathscr{S}$-morphism and also homomorphism, so it is a $\mathscr{G}$-morphism. This is easily seen to give an equivalence $[C, F, H]_{\tau} \rightarrow\left[C,(C \cdot H)_{\eta_{0}}, H\right]$. As remarked above, this shows that the Baer action is faithful.

Now we turn to showing that the action is transitive. For this let $\left[A, E_{1}, H\right]_{\sigma_{1}}$ and $\left[A, E_{2}, H\right]_{\sigma_{2}}$ represent two elements of $\operatorname{Ext}(H, A, \eta)$. We must produce an extension $[C, F, H]_{\tau}$ inducing $\eta_{0}$ and an equivalence isomorphism between $[C, F, H]_{\tau} \boxplus\left[A, E_{1}, H\right]_{\sigma_{1}}$ and $\left[A, E_{2}, H\right]_{\sigma_{2}}$.

Let $D$ denote the set of all pairs $\left(e_{1}, e_{2}\right)$ in $E_{1} \times E_{2}$ for which $\sigma_{1}\left(e_{1}\right)=\sigma_{2}\left(e_{2}\right)$ and $e_{1} a e_{1}^{-1}=e_{2} a e_{2}^{-1}$ for all $a \in A$. The set $D^{\prime}$ of all pairs $\left(e_{1}, e_{2}\right)$ in $E_{1} \times E_{2}$ satisfying the second condition is closed, being the intersection of the inverse images of 1 under the $\mathscr{S}$-morphisms $E_{1} \times E_{2} \rightarrow A$ sending $\left(e_{1}, e_{2}\right)$ to $e_{1} a e_{1}^{-1} e_{2} a^{-1} e_{2}^{-1}$ for the various $a \in A$. So $D=D^{\prime} \cap\left(E_{1} \times E_{2}\right)_{\sigma_{1}, \sigma_{2}}$ is closed in $E_{1} \times E_{2}$, and it is clearly a subgroup. Let $A_{0}$ denote the set of all elements $(a, a)$ with $a \in A$. Then $A_{0}$ is a closed subgroup of $A \times A$, which is a closed subgroup of $E_{1} \times E_{2}$, so $A_{0}$ is closed in $E_{1} \times E_{2}$ and so also in $D$. Also, $A_{0}$ is a normal subgroup of $D$. Write $F$ for $D / A_{0}$. The map $c \rightarrow(c, 1) A_{0}$ is a $\mathscr{G}$-isomorphism of $C$ onto a closed normal subgroup of $F$. Let $\tau: F \rightarrow H$ be the $\mathscr{G}$-morphism induced by the restriction to $D$ of the $\mathscr{G}$-morphism $\left(E_{1} \times E_{2}\right)_{\sigma_{2}, \sigma_{2}} \rightarrow H$ sending $\left(e_{1}, e_{2}\right)$ to $\sigma_{1}\left(e_{1}\right)=\sigma_{2}\left(e_{2}\right)$. We claim that $\tau$ is surjective. For this, let $h \in H$. Choose $\left(e_{1}, e_{2}\right) \in$ $\left(E_{1} \times E_{2}\right)_{\sigma_{1}, \sigma_{2}}$ with $\sigma_{1}\left(e_{1}\right)=\sigma_{2}\left(e_{2}\right)=h$. Then the conjugations by $e_{1}$ and $e_{2}$ on $A$ differ by an inner automorphism of $A$, say $e_{2} a e_{2}^{-1}=$ $e_{1} a_{0} a a_{0}^{-1} e_{1}^{-1}$ for all $a \in A$, with $a_{0}$ some element of $A$. Replacing $e_{1}$ by $e_{1} a_{0}$, we have $\left(e_{1}, e_{2}\right) \in D$ and $\tau\left(\left(e_{1}, e_{2}\right) A_{0}\right)=h$, showing that $\tau$ is surjec- 
tive. If $\left(e_{1}, e_{2}\right) \in D$ and $\sigma_{1}\left(e_{1}\right)=1$, then $e_{1} \in A$ so also $e_{2} \in A$ and $e_{2}^{-1} e_{1} \in C$. But then $\left(e_{1}, e_{2}\right) A_{0} \in C$. So $C$ is equal to the kernel of $\tau$ and we have an extension $[C, F, H]_{r}$, which is casily seen to induce $\eta_{0}$.

Finally, we obtain an equivalence isomorphism $[C, F, H]_{\text {. }} \boxplus$ $\left[A, E_{1}, H\right]_{\sigma_{1}} \rightarrow\left[A, E_{2}, H\right]_{\sigma_{2}}$ as follows: The $\mathscr{P}$-morphism $\left(D \times E_{1}\right)_{\lambda, \sigma_{1}} \rightarrow$ $E_{2}$, where $\lambda\left(e_{1}, e_{2}\right)=\sigma_{1}\left(e_{1}\right)=\sigma_{2}\left(e_{2}\right)$ for $\left(e_{1}, e_{2}\right) \in D$, given by $\left(e_{1}, e_{2}, \bar{e}_{1}\right) \mapsto$ $e_{2} e_{1}^{-1} \bar{e}_{1}$ is easily seen to be a homomorphism, i.e., a $\mathscr{G}$-morphism (we note that $e_{1}^{-1} \bar{e}_{1} \in A$ because $\left.\sigma_{1}\left(e_{1}\right)=\sigma_{1}\left(\bar{e}_{1}\right)\right)$. It is constant on the cosets mod the closed normal subgroup $A_{0} \times 1$ of $\left(D \times E_{1}\right)_{\lambda, \sigma_{1}}$. It therefore induces a $\mathscr{G}$-morphism $\left(D \times E_{1}\right)_{2, \sigma_{1}} /\left(A_{0} \times 1\right) \rightarrow E_{2}$, i.e., $\left(D / A_{0} \times E_{1}\right)_{\tau, \sigma_{1}} \rightarrow E_{2}$. The kernel of this map contains $C^{0}$, so it induces a $\mathscr{C}$-morphism $\left(F \times E_{1}\right)_{\tau, \sigma_{1}} / C^{0} \rightarrow E_{2}$, which is easily seen to give the desired equivalence. This completes the proof of the proposition.

Next we wish to admit some of the results of [3] which have to do with the functorial properties of Ext. We will exhibit the results we need and those definitions which are not obvious, and omit the proofs.

First, suppose that $G, A, B$, and $C$ are objects of $\mathscr{C}$, with $A, B$, and $C$ abelian. Suppose that the homomorphisms $\eta: G \rightarrow \operatorname{Aut}_{\mathscr{G}}(A)$, $\mu: G \rightarrow \operatorname{Aut}_{\mathscr{G}}(B)$, and $\nu: G \rightarrow \operatorname{Aut}_{\mathscr{G}}(C)$ make $A, B$, and $C$ into $G$-groups. Suppose further that

$$
1 \longrightarrow A \stackrel{\alpha}{\longrightarrow} B \stackrel{\beta}{\longrightarrow} C \longrightarrow 1
$$

is a short exact sequence of $\mathscr{C}$-morphisms and that we have

$$
\mu(g)\{\alpha(a)\}=\alpha \cdot(\eta(g)\{a\})
$$

and

$$
\nu(g)\{\beta(b)\}=\beta(\mu(g)\{b\})
$$

for all $a$ in $A, b$ in $B$, and $g$ in $G$. In this situation we shall say that $1 \rightarrow A \rightarrow B \rightarrow C \rightarrow 1$ is an exact sequence of $G$-groups (which happen to be abelian). It was shown in [3] that we have an exact sequence of homomorphisms

$$
\begin{aligned}
H^{1}(G, A) & \longrightarrow H^{1}(G, B) \longrightarrow H^{1}(G, C) \\
& \longrightarrow \operatorname{Ext}(G, A) \longrightarrow \operatorname{Ext}(G, B) \longrightarrow \operatorname{Ext}(G, C)
\end{aligned}
$$

(where we suppress $\eta, \mu$, and $\nu$ in the notation). The $H^{\prime}$ 's are the (obvious) groups of equivalence classes of those 1-cocycles which are also $\mathscr{S}$-morphisms.

To define the map $H^{1}(G, C) \rightarrow \operatorname{Ext}(G, A)$, suppose that $f: G \rightarrow C$ is a 1-cocycle and also an $\mathscr{P}$-morphism. Denote by $E_{f}$ the set of all elements $(b, g)$ of the semidirect product $(B \cdot G)_{\mu^{\prime}}$ for which $\beta(b)=$ 
$f(g)$. Then $E_{f}$ is a closed subgroup of $(B \cdot G)_{\mu}$. The restriction $\pi$ to $E_{f}$ of the canonical $\mathscr{G}$-morphism $(B \cdot G)_{\mu} \rightarrow G$ is surjective, and its kernel coincides with the image of $A$ under the injective $\mathscr{G}$ morphism $A \rightarrow(B \cdot G)_{\mu}$ sending each $a$ in $A$ to $(\alpha(a), 1)$. This gives an extension $\left[A, E_{f}, G\right]_{\pi}$ which, in fact, induces $\eta$. After several verifications, one sees that this defines a homomorphism $H^{1}(G, C) \rightarrow$ $\operatorname{Ext}(G, A)$.

To define the maps $\operatorname{Ext}(G, A) \rightarrow \operatorname{Ext}(G, B)$ and $\operatorname{Ext}(G, B) \rightarrow$ Ext $(G, C)$, suppose, more generally, that $M$ and $N$ are two abelian $G$-groups, say via homomorphisms $\delta: G \rightarrow$ Aut $(M)$ and $\varepsilon: G \rightarrow$ $\operatorname{Aut}_{\mathscr{S}}(N)$. Suppose that $\lambda: M \rightarrow N$ is a morphism of $G$-groups. If $[M, E, G]_{\pi}$ is an extension inducing $\delta$, denote by $M^{0}$ the set of all elements $\left(\lambda(m), m^{-1}\right)$ of the semidirect product $(N \cdot E)_{\varepsilon \pi}$ where $m \in M$. Then $M^{0}$ is a closed normal subgroup of $(N \cdot E)_{\varepsilon \pi}$, and we denote $(N \cdot E)_{\varepsilon \pi} / M^{0}$ by $E_{\lambda}$. We denote by $\pi_{\lambda}$ the surjective $\mathscr{G}$-morphism $E_{\lambda} \rightarrow G$ for which $\pi_{\lambda}\left((n, e) M_{0}\right)=\pi(e)$ for every $n$ in $N$ and $e$ in $E$. The map $n \mapsto(n, 1) M^{0}$ is then a $\mathscr{G}$-isomorphism of $N$ onto the kernel of $\pi_{\lambda}$ and this gives an extension $\left[N, E_{\lambda}, G\right]_{\pi_{\lambda}}$ called the kernel shift of $[M, E, G]_{\pi}$ with respect to $\lambda$. One easily verifies that $\left[N, E_{\lambda}, G\right]_{\pi_{\lambda}}$ induces $\varepsilon$ and that this gives a homomorphism $\operatorname{Ext}(G, M, \delta) \rightarrow$ $\operatorname{Ext}(G, N, \varepsilon)$.

We summarize the assertions, for later reference, in a proposition.

Proposition 2.2.1. Given a short exact sequence

$$
1 \longrightarrow A \longrightarrow B \longrightarrow C \longrightarrow 1
$$

of morphisms of abelian G-groups, the sequence

$$
\begin{aligned}
H^{1}(G, A) & \longrightarrow H^{1}(G, B) \longrightarrow H^{1}(G, C) \\
& \longrightarrow \operatorname{Ext}(G, A) \longrightarrow \operatorname{Ext}(G, B) \longrightarrow \operatorname{Ext}(G, C)
\end{aligned}
$$

described above is exact.

\section{Proof. See [3].}

Next we examine the variance of Ext in its other argument. Suppose that $G$ and $V$ are objects of $\mathscr{G}, V$ abelian, and that $\eta: G \rightarrow$ $\operatorname{Aut}_{S}(V)$ makes $V$ into a $G$-group (we shall not need the more general treatment given in [3] for arbitrary $V$, not necessarily abelian). Let $K$ be a closed normal subgroup of $G$. Denote by $V^{K}$ the set of all elements of $V$ which are left fixed by each $\eta(k)$ with $k \in K$. Then $V^{K}$ is a closed subgroup of $V$, being the intersection of inverse images of 1 under the $\mathscr{S}$-morphisms $V \rightarrow V$ sending $v$ to $\eta(k)\{v\} v^{-1}$ for the various $k$ in $K$. It was shown in [3] that we 
have an exact sequence of homomorphisms

$$
\begin{aligned}
(0) & \longrightarrow H^{1}\left(G / K, V^{K}\right) \longrightarrow H^{1}(G, V) \longrightarrow H^{1}(K, V)^{G} \\
& \longrightarrow \operatorname{Ext}\left(G / K, V^{K}\right) \longrightarrow \operatorname{Ext}_{K}(G, V) \longrightarrow H^{1}\left(G / K, H^{1}(K, V)\right) .
\end{aligned}
$$

Again we suppress in the notation the actions of $K, G$, and $G / K$ on various groups, it being understood that all of these arise naturally from the action of $G$ on $V$. If we denote by $Z^{1}(K, V)$ the abelian group of all 1-cocycle $\mathscr{S}$-morphisms $K \rightarrow V$, then $G$ operates on $Z^{1}(K, V)$ by $(g \cdot f)(k)=\eta(g)\left\{f\left(g^{-1} k g\right)\right\}=g \cdot f\left(g^{-1} k g\right)$. This action stabilizes the group $B^{1}(K, V)$ of 1-coboundary $\mathscr{S}$-morphisms $K \rightarrow V$, so induces an action of $G$ on $H^{1}(K, V)$, and $H^{1}(K, V)^{G}$ denotes the fixed part of $H^{1}(K, V)$ with respect to this action.

The first two maps $H^{1}\left(G / K, V^{K}\right) \rightarrow H^{1}(G, V) \rightarrow H^{1}(K, V)^{G}$ are just the usual inflation and restriction.

To define the map $H^{1}(K, V)^{G} \rightarrow \operatorname{Ext}\left(G / K, V^{K}\right)$ which is called the transgression, let $f \in Z^{1}(K, V)$. Write $V \cdot K$ for $(V \cdot K)_{\eta \mid K}$, and denote by $K_{f}$ the set of all elements $(f(k), k)$ of $V \cdot K$ where $k \in K$. Denote by $N_{f}$ the normalizer of $K_{f}$ in $V \cdot G=(V \cdot G)_{\eta}$. Then both $K_{f}$ and $N_{f}$ are closed subgroups of $V \cdot G$. Now one can show that the restriction to $N_{f}$ of the canonical $\mathscr{G}$-morphism $V \cdot G \rightarrow G$ is surjective if and only if the class of $f$ in $H^{1}(K, V)$ is $G$-fixed. Assuming it is, this surjection induces a surjective $\mathscr{G}$-morphism $\delta_{f}: N_{f} / K_{f} \rightarrow G / K$. The $\mathscr{G}$-isomorphism $V^{K} \rightarrow V \cdot G$ sending each $v$ in $V^{K}$ to $(v, 1)$ is easily seen to take values in $N_{f}$, so it induces a $\mathscr{G}$ morphism $V^{K} \rightarrow N_{f} / K_{f}$. One jverifies that this is injective and that it identifies $V^{K}$ with the kernel of $\delta_{f}$, so that we have an extension $\left[V^{K}, N_{f} / K_{f}, G / K\right]_{\delta_{f}}$, which induces the same $G / K$-group structure on $V^{K}$ as is naturally induced by $\eta$. After many verifications, one sees that this defines a homomorphism $H^{1}(K, V)^{G} \rightarrow \operatorname{Ext}\left(G / K, V^{K}\right)$.

To define the map $\operatorname{Ext}\left(G / K, V^{K}\right) \rightarrow \operatorname{Ext}_{K}(G, V)$, whose range is the set of equivalence classes of extensions of $V$ by $G$ inducing $\eta$ which are $K$-split, let $\left[V^{K}, E, G / K\right]_{\sigma}$ be an extension inducing that action which is naturally induced by $\eta$. We lift this to the extension $\left[V^{K}, E^{\prime}, G\right]_{\pi}$, where $E^{\prime}$ is the fiber product $E \times_{\sigma, \tau} G, \tau: G \rightarrow G / K$ the canonical map, and $\pi^{\prime}(e, g)=g$ for all $(e, g)$ in $E^{\prime}$. Then we apply the kernel shift with respect to the morphism $V^{K} \rightarrow V$ of $G$ groups, obtaining the extension $\left[V, E^{\prime \prime}, G\right]_{\pi^{\prime \prime}}$ where $E^{\prime \prime}$ is the quotient of $\left(V \cdot E^{\prime}\right)_{\eta_{\pi^{\prime}}}$ by the closed normal subgroup $\left(V^{K}\right)^{0}$ consisting of all elements $\left(v, v^{-1}\right)$ where $v \in V^{K}$. The verifications here are very easy, and show that this defines a homomorphism $\operatorname{Ext}\left(G / K, V^{K}\right) \rightarrow \operatorname{Ext}(G, V)$ which actually takes values in $\operatorname{Ext}_{K}(G, V)$.

Finally, to define the map $\operatorname{Ext}_{K}(G, V) \rightarrow H^{1}\left(G / K, H^{1}(K, V)\right)$, and describe what its range is, let $[V, E, G]_{\pi}$ be an extension inducing 
$\eta$ and suppose that it is $K$-split, i.e., that there is a $\mathscr{G}$-morphism $\lambda: K \rightarrow E$ such that $\pi(\lambda(k))=k$ for every $k$ in $K$. Then, using $\lambda$, we may identify the inverse image of $K$ in $E$ with $V \cdot K$ so that $\pi(v, k)=k$ for $v \in V, k \in K$. Since this is a closed normal subgroup of $E$, we have an $E$-group structure $\gamma: E \rightarrow \operatorname{Aut}_{\varphi}(V \cdot K)$ on $V \cdot K$ by conjugations within $E$. We also have a $G$-group structure $\rho$ : $G \rightarrow \operatorname{Aut}_{\mathscr{G}}(V, K)$ where $\rho(g)\{(v, k)\}=\left(\eta(g)\{v\}, g k g^{-1}\right)$ for $v \in V, k \in K$, and $g \in G$. We define a function $f: E \rightarrow \operatorname{Aut}_{\mathscr{c}}(V \cdot K)$ by $f(e)=$ $\gamma(e) \circ \rho\left(\pi\left(e^{-1}\right)\right)$ for $e$ in $E$. Then in fact $f(e)$ belongs to the subgroup $A$ of $\operatorname{Aut}_{\mathscr{G}}(V \cdot K)$ consisting of all $\mathscr{G}$-automorphisms which induce the identity automorphism on both $V$ and $(V \cdot K) / V$. We denote by $V^{\sharp}$ the subgroup of $A$ consisting of all conjugations on $V \cdot K$ by elements of $V$, and then $A / V^{\sharp}$ is naturally isomorphic with the group $H^{1}(K, V)$. If $A$ is made into an (abstract) $E$-module by $e \cdot \psi=\rho(\pi(e)) \circ \psi \circ \rho\left(\pi\left(e^{-1}\right)\right)$ for $e \in E, \psi \in A$, then $f: E \rightarrow A$ is in fact a 1-cocycle. The action of $E$ on $A / V^{\#}$, induced by that of $E$ on $A$, factors through $G$ via $\pi$, and this coincides with the usual action of $G$ on $H^{1}(K, V)$. This, in turn, factors through $G / K$ via $\tau$. Now one puts $g(e)=f(e) V^{\sharp}$ so $g: E \rightarrow A / V^{\sharp}=H^{1}(K, V)$, and then $g$ is a 1-cocycle. This, in turn, factors through $G / K$, inducing a 1-cocycle $h: G / K \rightarrow H^{1}(K, V)$. The map $\operatorname{Ext}_{K}(G, V) \rightarrow H^{1}\left(G / K, H^{1}(K, V)\right)$ sends the class of $[H, E, G]_{\pi}$ to the class of $h$. One must beware that, although $H^{1}(K, V)$ consists of equivalence classes of 1-cocycle $\mathscr{S}$ morphisms, it does not in general carry a structure of object of $\mathscr{S}$ and $H^{1}\left(G / K, H^{1}(K, V)\right)$ consists of equivalence classes of arbitrary 1-cocycles $G / K \rightarrow H^{1}(K, V)$. We summarize this for later reference in a proposition.

Proposition 2.2.2. Let $V, G$, and $K$ be objects of $\mathscr{G}$ where $K$ is a closed normal subgroup of $G$ and $V$ is an abelian G-group. Then we have an exact sequence of abelian group homomorphisms.

$$
\begin{aligned}
(0) & \longrightarrow H^{1}\left(G / K, V^{K}\right) \longrightarrow H^{1}(G, V) \longrightarrow H^{1}(K, V)^{G} \\
& \longrightarrow \operatorname{Ext}\left(G / K, V^{K}\right) \longrightarrow \operatorname{Ext}_{K}(G, V) \longrightarrow H^{1}\left(G / K, H^{1}(K, V)\right)
\end{aligned}
$$

where the group $H^{1}(K, V)$ consists of equivalence classes of 1-cocycle $\mathscr{S}$-morphisms while $H^{1}\left(G / K, H^{1}(K, V)\right)$ consists of equivalence classes of arbitrary 1-cocycles.

\section{Proof. See [3].}

Next we examine how (parts of) the exact sequences of Propositions 2.2.1 and 2.2.2 fit together with the situation of Proposition 2.1.

First suppose that $A, B$, and $G$ are objects of $\mathscr{G}$ and that 
$\alpha: A \rightarrow B$ is a $\mathscr{G}$-morphism. Suppose that $\eta: G \rightarrow O(A)$ and $\mu: G \rightarrow$ $O(B)$ are homomorphisms. Let $A_{1}$ and $B_{1}$ denote the centers of $A$ and $B$, respectively, and suppose that $\alpha\left(A_{1}\right) \cong B_{1}$. Let $\eta_{0}: G \rightarrow$ Aut $\left(A_{1}\right)$ and $\mu_{0}: G \rightarrow$ Aut $\left(B_{1}\right)$ be induced by $\eta$ and $\mu$ and suppose that these make $A_{1}$ and $B_{1}$ into $G$-groups. Suppose also that the restriction of $\alpha$ to $A_{1}$ is a morphism of $G$-groups so that it yields a homomorphism $\operatorname{Ext}\left(G, A_{1}\right) \rightarrow \operatorname{Ext}\left(G, B_{1}\right)$ of abelian groups.

Now suppose that $[A, E, G]_{\pi}$ is an extension inducing $\eta$. This gives rise to an $E$-group structure $\gamma: E \rightarrow \operatorname{Aut}_{g}(A)$ on $A$ by conjugations within $E$. Suppose we are given an $E$-group structure $\delta: E \rightarrow$ $\operatorname{Aut}_{\mathscr{G}}(B)$ such that

$$
\begin{array}{ll}
\delta(e)\{\alpha(a)\}=\alpha(\gamma(e)\{a\}) & \text { for } e \text { in } E, a \text { in } A \\
\delta(a)\{b\}=\alpha(a) b \alpha(a)^{-1} & \text { for } a \text { in } A, b \text { in } B
\end{array}
$$

and such that $\delta$ induces the homomorphism $\mu: G \rightarrow O(B)$. Then we may construct an extension $[B, M, G]_{\sigma}$ inducing $\mu$ and making a commutative diagram

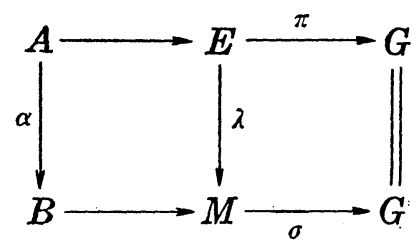

as follows: Let $A^{0}$ denote the subset of $(B \cdot E)_{\delta}$ consisting of all elements $\left(\alpha(\alpha), \alpha^{-1}\right)$ with $a$ in $A$. Then $A^{0}$ is a closed normal subgroup of $(B \cdot E)_{j}$, and we put $M=(B \cdot D)_{i} / A^{0}$. The $\mathscr{G}$-morphism $(B \cdot E)_{\dot{o}} \rightarrow G$ sending each $(b, e)$ to $\pi(e)$ induces a surjective $\mathscr{G}$-morphism $\sigma: M \rightarrow G$. The $\mathscr{G}$-morphism $B \rightarrow M$ sending each $b$ in $B$ to $(b, 1) A^{0}$ is a $\mathscr{G}$-isomorphism of $B$ onto the kernel of $\sigma$. This gives an extension $[B, M, G]$ which is easily seen to induce $\mu$. We define $\lambda: E \rightarrow M$ by $\lambda(e)=(1, e) A^{0}$ for $e \in E$, and then $\lambda$ is a $\mathscr{G}$-morphism making the above diagram commute. We call $[B, M, G]_{\sigma}$ the kernel shift of $[A, E, G]_{\pi}$ along $\alpha$ and with respect to $\delta$.

Suppose $[A, E, G]_{\pi}, \alpha$, and $\delta$ are as above, and $[B, M, G]_{\sigma}$ is the kernel shift as above. We wish to show that for any extension of $A_{1}$ by $G$ inducing $\eta_{0}$, we may operate with this extension on $[A, E, G]_{\pi}$ and take a kernel shift or we may take kernel shifts of both $[A, E, G]_{\pi}$ and this extension of $A_{1}$ by $G$ and then operate, and thereby obtain equivalent extensions of $B$ by $G$.

Let $\left[A_{1}, P, G\right]_{\varepsilon}$ be an extension inducing $\eta_{0}$. We write $\left[A, E^{\prime}, G\right]_{\pi^{\prime}}=$ $\left[A_{1}, P, G\right]_{\varepsilon} \boxplus[A, E, G]_{\pi}$, where $E^{\prime}=(P \times E)_{\varepsilon, \pi} / A_{1}^{0}$ and $\pi^{\prime}\left((p, e) A_{1}^{0}\right)=\pi(e)$ for all $p$ in $P, e$ in $E$, and the map $A \rightarrow E^{\prime}$ sends each $\alpha$ in $A$ to 
$(1, a) A_{1}^{0}$. Define $\gamma^{\prime}: E^{\prime} \operatorname{Aut}_{\mathscr{S}}(A)$ by $\gamma^{\prime}\left((p, e) A_{1}^{0}\right)=\gamma(e)$, which is a definition because $A_{1}$ is contained in the kernel of $\gamma$. Then one easily sees that $\gamma^{\prime}$ makes $A$ into an $E^{\prime}$-group. Next define $\delta^{\prime}: E^{\prime} \rightarrow \operatorname{Aut}_{\mathscr{Q}}(B)$ by $\delta^{\prime}\left((p, e) A_{1}^{0}\right)=\delta(e)$, also a definition because $A_{1}$ is contained in the kernel of $\delta$. One easily sees that we have

$$
\begin{array}{ll}
\delta^{\prime}\left(e^{\prime}\right)\{\alpha(a)\}=\alpha\left(\gamma^{\prime}\left(e^{\prime}\right)\{a\}\right) & \text { for } e^{\prime} \text { in } E^{\prime}, a^{\prime} \text { in } A \\
\delta^{\prime}(a)\{b\}=\alpha(a) b \alpha(a)^{-1} & \text { for } a \text { in } A, b \text { in } B
\end{array}
$$

and that $\delta^{\prime}$ induces $\mu: G \rightarrow O(B)$, while $\gamma^{\prime}$ induces $\eta: G \rightarrow O(A)$. So we may consider the kernel shift of $\left[A, E^{\prime}, G\right]_{\pi^{\prime}}$ along $\alpha$ with respect to $\delta^{\prime}$. Let us denote this by $\left[B, M^{\prime}, G\right]_{\sigma^{\prime}}$.

On the other hand, we may take the kernel shift of $\left[A_{1}, P, G\right]_{s}$ along $\alpha_{\mid A_{1}}: A_{1} \rightarrow B_{1}$. . Let us denote this by $\left[B_{1}, Q, G\right]_{\varepsilon_{1}}$, where $Q=$ $\left(B_{1} \cdot P\right)_{u_{0} \varepsilon} / A_{1}^{0}, \cdots$ etc.

Proposition 2.3. In the notation introduced above, the two extensions $\left[B_{1}, Q, G\right]_{\varepsilon_{1}} \boxplus[B, M, G]_{\sigma}$ and $\left[B, M^{\prime}, G\right]_{\sigma^{\prime}}$ are equivalent.

Proof. For notational convenience, we will write $X \times_{\xi, \rho} Y$ rather than $(X \times Y)_{\xi, \rho}$ for a fiber product of $X$ and $Y$ with respect to maps $\xi$ and $\rho$ of $X$ and $Y$, respectively, into some third space, both here and subsequently. We must produce an equivalence $\mathscr{G}$-isomorphism

$$
\left[\left(B_{1} \cdot P\right)_{\mu_{0} \varepsilon} / A_{1}^{0}\right] \times_{\varepsilon_{1}, \sigma}\left[(B \cdot E)_{\hat{o}} / A^{0}\right] / B_{1}^{0} \longrightarrow\left(B \cdot\left(P \mathrm{X}_{\varepsilon, \pi} E / A_{1}^{0}\right)\right)_{\delta^{\prime}} / A^{0} .
$$

Using the assumptions on $\mathscr{C}$ in a straightforward manner, this map may be constructed by the recipe $\left(\left(b_{1}, p\right) A_{1}^{0},(b, e) A^{0}\right) B_{1}^{0} \rightarrow\left(b_{1} b,(p, e) A_{1}^{0}\right) A^{0}$ where $b_{1} \in B_{1}, p \in P, b \in B$, and $e \in E$.

Now suppose $A$ and $G$ are objects of $\mathscr{C}$ and $\eta: G \rightarrow O(A)$ is a homomorphism. Let $C$ denote the center of $A$ and $\eta_{0}: G \rightarrow \operatorname{Aut}(C)$ the homomorphism induced by $\eta$. Suppose that $\eta_{0}(G) \cong \operatorname{Aut}_{\mathscr{S}}(C)$ and that $\eta_{0}$ makes $C$ into a $G$-group. Let $H$ be an object of $\mathscr{G}$ and $\omega: H \rightarrow G$ a $\mathscr{G}$-morphism (not necessarily injective or surjective). Then we have a group homomorphism $\operatorname{Ext}\left(G, C, \eta_{0}\right) \rightarrow \operatorname{Ext}\left(H, C, \eta_{0} \omega\right)$ and a set map $\operatorname{Ext}(G, A, \eta) \rightarrow \operatorname{Ext}(H, A, \eta \omega)$, although the set $\operatorname{Ext}(G, A, \eta)$ may be empty.

Suppose $[A, E, G]_{\pi}$ is an extension inducing $\eta$. Denote $E \mathbf{X}_{\pi, \omega} H$ by $E^{\omega}$ and put $\pi^{\omega}(e, h)=h$ for all $(e, h) \in E^{\omega}$, so the map $\operatorname{Ext}(G, A, \eta) \rightarrow$ $\operatorname{Ext}(H, A, \eta \omega)$ sends the class of $[A, E, G]_{\pi}$ to that of $\left[A, E^{\omega}, H\right]_{\pi^{\omega}}$.

Now suppose $[C, M, G]_{\sigma}$ is an extension inducing $\eta_{0}$. Put $M^{\omega}=$ $M \times_{\sigma, \omega} H$ and $\sigma^{\omega}(m, h)=h$ for all $(m, h) \in M^{\omega}$, so the homomorphism $\operatorname{Ext}\left(G, C, \eta_{0}\right) \rightarrow \operatorname{Ext}\left(H, C, \eta_{0} \omega\right)$ sends the class of $[C, M, G]_{0}$ to that 
of $\left[C, M^{\omega}, H\right]_{o \omega}$.

Next put $E_{1}=\left(M \times_{\sigma, \pi} E\right) / C^{0}$ and $\pi_{1}\left((m, e) C^{0}\right)=\sigma(m)=\pi(e)$ for $(m, e) C^{0} \in E_{1}$ (well-defined because $C$ is contained in the kernel of both $\sigma$ and $\pi$ ). Then we have $\left[A, E_{1}, G\right]_{\pi_{1}}=[C, M, G]_{\sigma} \boxplus[A, E, G]_{\pi}$. Put $E_{1}^{\omega}=E_{1} \mathbf{X}_{\pi_{1}, \omega} H$ and $\pi_{1}^{\omega}\left(e_{1}, h\right)=h$ for all $\left(e_{1}, h\right) \in E_{1}^{\omega}$. Then the map $\operatorname{Ext}(G, A, \eta) \rightarrow \operatorname{Ext}(H, A, \eta \omega)$ sends the class of $\left[A, E_{1}, G\right]_{\pi_{1}}$ to that of $\left[A, E_{1}^{i}, H\right]_{\pi_{1} \omega}$.

Proposition 2.4. In the notation introduced above, the two extensions $\left[C, M^{\omega}, H\right]_{\sigma^{\omega}} \boxplus\left[A, E^{\omega}, H\right]_{\pi^{\omega}}$ and $\left[A, E_{1}^{\omega}, H\right]_{\pi_{1} \omega}$ are equivalent.

Proof. We need an equivalence $\mathscr{G}$-isomorphism

$$
\left(M \mathrm{X}_{\sigma, \omega} H\right) \mathbf{X}_{\sigma^{(0,}, \pi^{\omega}}\left(E \mathbf{X}_{\pi, \omega} H\right) /_{\sigma^{0}} \rightarrow\left(\left(M \mathbf{X}_{\sigma, \pi} E\right) / C^{0}\right) \mathbf{X}_{\pi_{1}, \omega} H
$$

which is obtainable from the recipe $\left(\left(m, h_{1}\right),\left(e, h_{2}\right)\right) C^{0} \rightarrow\left((m, e) C^{0}, h_{1} h_{2}\right)$ where $m \in M, e \in E, h_{1}, h_{2} \in H$.

Proposition 2.5. Suppose $A$ and $G$ are objects of $\mathscr{G}, A$ is abelian, and the homomorphism $\eta: G \rightarrow$ Aut $_{(A)}$ makes $A$ into a G-group. Suppose $A$ has closed subgroups $A_{1}$ and $A_{2}$ both of which are stable under $\eta(G)$ and such that $A$ is equal to the direct product $A_{1} \times A_{2}$. Then we have an isomorphism $\operatorname{Ext}(G, A, \eta) \cong \operatorname{Ext}\left(G, A_{1}, \eta_{1}\right) \times$ $\operatorname{Ext}\left(G, A_{2}, \eta_{2}\right)$, where $\eta_{i}: G \rightarrow \operatorname{Aut}_{S}\left(A_{i}\right)$ is the homomorphism induced by $\eta$ and taking restrictions, for $i=1,2$.

Proof. By Proposition 2.2.1, the short exact sequeuces

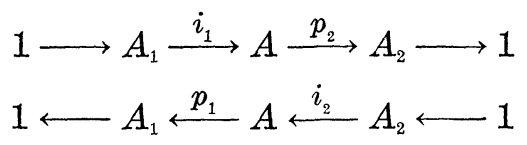

of abelian $G$-groups give rise to exact sequences

$$
\begin{aligned}
& \operatorname{Ext}\left(G, A_{1}, \eta_{1}\right) \stackrel{\alpha}{\longleftrightarrow} \operatorname{Ext}(G, A, \eta) \stackrel{\beta}{\longrightarrow} \operatorname{Ext}\left(G, A_{2}, \eta_{2}\right) \\
& \operatorname{Ext}\left(G, A_{1}, \eta_{1}\right) \stackrel{\beta}{\longleftarrow} \operatorname{Ext}(G, A, \eta) \stackrel{\delta}{\longleftarrow} \operatorname{Ext}\left(G, A_{2}, \eta_{2}\right) .
\end{aligned}
$$

One easily sees that $\beta \circ \alpha$ and $\gamma \circ \delta$ are equal to the identity maps on $\operatorname{Ext}\left(G, A_{1}, \eta_{1}\right)$ and $\operatorname{Ext}\left(G, A_{2}, \eta_{2}\right)$, respectively. For example, if $\left[A_{1}, E_{1}, G\right]_{\pi_{1}}$ induces $\eta_{1}$, then $\beta \circ \alpha$ sends its class to that of the extension $\left[A_{1},\left(A_{1} \cdot\left(\left(A \cdot E_{1}\right)_{\eta_{\pi_{1}}} / A_{1}^{0}\right)\right)_{\eta_{1} \pi} / A^{0}, G\right]_{\pi^{\prime}}$ where $\pi:\left(A \cdot E_{1}\right)_{\eta_{\pi_{1}}} / A_{1}^{0} \rightarrow G$ sends $\left(a, e_{1}\right) A_{1}^{0}$ to $\pi_{1}\left(e_{1}\right)$. An equivalence $\mathscr{G}$-isomorphism of this to the extension $\left[A_{1}, E_{1}, G\right]_{\pi_{1}}$ is obtainable from the recipe $\left(a_{1},\left(a, e_{1}\right) A_{1}^{0}\right) A^{0} \rightarrow$ $a_{1} p_{1}(a) e_{1}$.

It follows that $\alpha$ and $\delta$ are injective and that $\beta$ and $\gamma$ are sur- 
jectiue. Now one sees immediately that the homomorphism

$$
\operatorname{Ext}\left(G, A_{1}, \eta_{1}\right) \times \operatorname{Ext}\left(G, A_{2}, \eta_{2}\right) \stackrel{\alpha \times \delta}{\longrightarrow} \operatorname{Ext}(G, A, \eta)
$$

is an isomorphism.

6. Character groups of pro-affine algebraic groups. Throughout this section we will assume that the field $F$ is algebraically closed, but we do not specify its characteristic. If $(G, P(G))$ is a pro-affine algebraic group over $F$, we denote by $\hat{G}$ the group of all rational homomorphisms $G \rightarrow F^{*}$ under valuewise multiplication. If $(H, P(H))$ is another pro-affine algebraic group over $F$ and $\alpha: G \rightarrow H$ a rational homomorphism, the map $\hat{\alpha}: \hat{H} \rightarrow \widehat{G}$ defined by $\hat{\alpha}(\phi)=\phi \circ \alpha$ for $\phi \in H$ is a homomorphism. These definitions make ${ }^{\wedge}$ a functor from the category of pro-affine algebraic groups over $F$ to the category of abelian groups.

An element of $\hat{G}$ is, in particular, a polynomial function $G \rightarrow F$, i.e., an element of $P(G)$. If $\gamma: P(G) \rightarrow P(G) \otimes P(G)$ denotes the comultiplication in $P(G)$, then $\hat{G}$ is precisely the set of group-like elements of $P(G)$, i.e., the set of all nonzero elements $\phi$ of $P(G)$ for which $\gamma(\phi)=\phi \otimes \phi$. If $\alpha: G \rightarrow H$ is as above, then $\hat{\alpha}$ is just the restriction to $\hat{H}$ of the Hopf algebra homomorphism $\alpha^{\prime}: P(H) \rightarrow P(G)$ induced by $\alpha$.

Because the group $F^{*}$ is reductive and abelian, the kernel of any rational homomorphism $G \rightarrow F^{*}$ contains the subgroup $G_{u} G^{\prime}$ of $G$ which is generated by the unipotent radical $G_{u}$ of $G$ and the algebraic hull $G^{\prime}$ of the commutator subgroup of $G . G_{u} G^{\prime}$ is a normal algebraic subgroup of $G$ and the canonical map $G \rightarrow G / G_{u} G^{\prime}$ is universal, in the obvious sence, among rational homomorphisms from $G$ into reductive abelian pro-affine algebraic groups over $F$. So we see that $\hat{G}$ is isomorphic with $\widehat{G / G_{u} G^{\prime}}$. It is therefore natural to consider the restriction of ${ }^{-}$to the full subcategory of all reductive abelian pro-affine algebraic groups over $F$. We will see that this restriction is a contravariant categorical equivalence onto the category of all abelian groups without $p$-torsion, where $p$ is the characteristic of $F$. If $p=0,{ }^{\wedge}$ is an equivalence onto the category of all abelian groups.

Suppose $(G, P(G))$ is a reductive abelian pro-affine algebraic group over $F$. Let $F[\hat{G}]$ denote the group algebra of $\hat{G}$ over $F$. It contains $\hat{G}$ as an $F$-basis, and multiplication is defined in the obvious way in terms of the group multiplication in $\hat{G}$. We make $F[\hat{G}]$ into a Hopf algebra defining the comultiplication $\gamma$, antipode $\eta$, and counit $c$ by 


$$
\begin{aligned}
\gamma\left(\sum f_{i} \phi_{i}\right) & =\sum f_{i} \phi_{i} \otimes \phi_{i} \\
\eta\left(\sum f_{i} \phi_{i}\right) & =\sum f_{i} \phi_{i}^{-1} \\
c\left(\sum f_{i} \phi_{i}\right) & =\sum f_{i} .
\end{aligned}
$$

The map $F[\hat{G}] \rightarrow P(G)$ obtained by extending the inclusion $\hat{G} \rightarrow P(G)$ by $F$-linearity is easily seen to be a Hopf algebra homomorphism. It is well-known that distinct homomorphisms from a group to the multiplicative group of a field are independent functions over that field, so the above map is injective.

To see that the above map is an isomorphism, we observe that, since $P(G)$ is a rational module for the reductive abelian group $G$, Schur's lemma implies that $P(G)$ is a sum of 1-dimensional $G$-submodules. If $F u$ is a 1 -dimensional $G$-submodule spanned by $u$, it is easy to verify that $(1 / u(e)) u$ belongs to $\hat{G}$, where $e$ denotes the identity element of $G$. This proves the following proposition.

Proposition 3.1. Let $(G, P(G))$ be a reductive abelian pro-affine algebraic group over $F$. Then the natural Hopf algebra homomorphism $F[\hat{G}] \rightarrow P(G)$ is an isomorphism.

Let $p$ denote the characteristic of $F$, and let $(G, P(G))$ be as in the above proposition. Then if $p \neq 0$, the abelian group $\hat{G}$ has no $p$-torsion. To see this we simply observe than if $\phi \in \widehat{G}$, then $\phi^{n}=1$ if and only if the image of $\phi$ is contained in the group of $n$th roots of unity in $F^{*}$ and the only $\left(p^{m}\right)$ th root of unity in $F^{*}$ is 1 for every positive integer $m$. This shows that ${ }^{\wedge}$ is a functor from the category of reductive abelian pro-affine algebraic groups over $F$ to the category of abelian groups without $p$-torsion.

Now we proceed to define a functor inverse to $\uparrow$, which we will denote by ${ }^{`}$. Let $X$ be any abelian group without $p$-torsion, or just any abelian group if $p=0$. Let $F[X]$ denote the group algebra of $X$ over $F$. We wish to show that $F[X]$ is reduced, i.e., that it contains no nonzero nilpotent elements.

If $p \neq 0$, let $\lambda=\sum f_{i} x_{i}$ be a nilpotent element of $F[X]$, where $f_{i} \in F$ and $x_{i} \in X$. Then $\lambda^{p^{k}}=0$ for some positive integer $k$. Now $\lambda^{p^{k}}=\sum f_{i}^{p^{k}} x_{i}^{p^{k}}$ and the $x_{i}^{p^{k}}$ are distinct because the homomorphism $X \rightarrow X$ sending each $x$ to $x_{i}^{p^{k}}$ is injective since $X$ has no $p$-torsion. So we must have that each $f_{i}^{p k}=0$, and hence each $f_{i}=0$, i.e., $\lambda=0$.

If $p=0$, again let $\lambda=\sum f_{i} x_{i}$ be a nilpotent element of $F[X]$. If $X^{\prime}$ is the subgroup of $X$ generated by the $x_{i}$ 's, we have a natural inclusion $F$-algebra homomorphism $F\left[X^{\prime}\right] \rightarrow F[X]$ and $\lambda \in F\left[X^{\prime}\right]$. So we may assume that $X$ is finitely generated. Then $X \cong A \times B$ where $A$ is a finite abelian group and $B$ is a product of finitely many copies of the integers. So we have an $F$-algebra isomorphism 
$F[X] \cong F[A] \bigotimes_{F} F[B]$. It is a classical result that $F[A]$ is a simisimple $F$-algebra and hence isomorphic with a direct $F$-algebra sum of finitely many copies of $F$, in fact $|A|$ copies. Thus $F[X]$ is a direct $F$-algebra sum of finitely many copies of $F[B]$. Now $F[B]$ is isomorphic with the $F$-subalgebra $F\left[x_{1}, x_{1}^{-1}, \cdots, x_{n}, x_{n}^{-1}\right]$ of the rational function field $F\left(x_{1}, \cdots, x_{n}\right)$, so it is an integral domain. This shows that $F[X]$ is reduced.

Now make $F[X]$ into a Hopf algebra, defining the comultiplication $\gamma$, antipode $\eta$, and counit $c$ by

$$
\begin{aligned}
& \gamma\left(\sum f_{i} x_{i}\right)=\sum f_{i} x_{i} \otimes x_{i} \\
& \eta\left(\sum f_{i} x_{i}\right)=\sum f_{i} x_{i}^{-1} \\
& c\left(\sum f_{i} x_{i}\right)=\sum f_{i} .
\end{aligned}
$$

If $\psi: F[X] \rightarrow F$ is any $F$-algebra homomorphism, its restriction to $X$ is a group homomorphism $X \rightarrow F^{*}$. Conversely, given any group homomorphism $X \rightarrow F^{*}$, its extension to $F[X]$ by $F$-linearity is an $F$-algebra homomorphism $F[X] \rightarrow F$. This correspondence gives a bijection from the set $\mathscr{C}(F[X])$ of all $F$-algebra homomorphisms $F[X] \rightarrow F$ to the set $\operatorname{Hom}\left(X, F^{*}\right)$. One easily checks that this bijection is a group homomorphism, where $\mathscr{G}(F[X])$ carries the group structure coming from the Hopf algebra structure of $F[X]$ and $\operatorname{Hom}\left(X, F^{*}\right)$ carries that of valuewise multiplication. We denote $\operatorname{Hom}\left(X, F^{*}\right)$ by $\check{X}$. Then by Theorem 2.1 of [6] and the above identification, we have that $(\check{X}, F[X])$ is the structure of a proaffine algebraic group over $F$ which is clearly abelian. If $x \in X$, $\sigma \in \check{X}$, then viewing $x$ as an element of $F[X]$, one easily checks that we have $\sigma \cdot x=\sigma(x) x$. So $F[X]$ is the sum of 1-dimensional stable submodules for the action of $\check{X}$ by left translations, and hence $(\check{X}, F[X])$ is a reductive abelian pro-affine algebraic group over $F$.

Suppose $\rho: X \rightarrow Y$ is a homomorphism of abelian groups without $p$-torsion. Then $\rho$ gives rise, uniquely, to an $F$-algebra homomorphism $F[X] \rightarrow F[Y]$ which is easily seen to be a homomorphism of Hopf algebras. One can check directly that under the above identifications, the induced homomorphism $\mathscr{G}(F[Y]) \rightarrow \mathscr{G}(F[X])$ corresponds to the natural homomorphism $\check{Y} \rightarrow \breve{X}$ sending a homomorphism $Y \rightarrow F^{*}$ to its composition with $\rho$. We denote this homomorphism $\check{Y} \rightarrow \check{X}$ by $\check{\rho}$. Now one easily checks that this makes ${ }^{`}$ a functor from the category of abelian groups without $p$-torsion to the category of reductive abelian pro-affine algebraic groups over $F$.

With Proposition 3.1, one can see immediately that the functor

is naturally equivalent to the identity functor on the category of reductive abelian pro-affine algebraic groups over $F$. 
To see that ${ }^{\circ}>$ is naturally equivalent to the identity functor on the category of abelian groups without $p$-torsion, one merely has to check that for an abelian group $X$ without $p$-torsion, the elements of $X$ are the only group-like elements of the Hopf algebra $F[X]$. But an element $\sum_{i=1}^{n} f_{i} x_{i}$ of $F[X]$ is group-like if and only if it is nonzero and $\sum_{i=1}^{n} f_{i} x_{i} \otimes x_{i}=\sum_{i, j=1}^{n} f_{i} f_{j} x_{i} \otimes x_{j}$. It is easily seen that this requires that all the $f_{i}$ 's are zero except one, which must equal 1 , So we have the following theorem.

THEOREM 3.2. The functor ${ }^{\wedge}$ is a contravariant categorical equivalence from the category of reductive abelian pro-affine algebraic groups over $F$ to the category of abelian groups without p-torsion, where $p$ is the characteristic of $F$. The functor ${ }^{\sim}$ is a two sided inverse to .

One easily sees that a reductive abelian pro-affine algebraic group $(G, P(G)$ ) over $F$ is affine (i.e., $P(G)$ is finitely generated as an $F$-algebra) if and only if the abelian group $\hat{G}$ is finitely generated. So our contravariant categorical equivalence carries the full subcategory of reductive abelian affine algebraic groups over $F$ onto the full subcategory of finitely generated abelian groups without $p$-torsion.

Also, one easily sees that a pro-affine group $(G, P(G)$ ) as above is connected if and only if $\hat{G}$ is torsion free, and that $G$ is totally disconnected, i.e., pro-finite, if and only if $\hat{G}$ is a torsion group. So if $(G, P(G))$ is as above and $G_{1}$ denotes the identity component of $G$, then the exact sequence

$$
1 \longrightarrow G_{1} \longrightarrow G \longrightarrow G / G_{1} \longrightarrow 1
$$

corresponds, via $\hat{\wedge}$, to the exact sequence

$$
1 \longleftarrow \widehat{G}_{1} \longleftarrow \hat{G} \longleftarrow \widehat{G / G_{1} \longleftarrow}
$$

and the map $\widehat{G / G_{1}} \rightarrow \widehat{G}$ is easily seen to map $\widehat{G / G_{1}}$ isomorphically onto the torsion subgroup of $\hat{G}$.

7. Applications. We may use the character theory to provide three counterexamples. We will see that there exists an extension [ $A$, $B, C]_{\pi}$ of (connected) pro-affine algebraic groups over $F$ for which there is no polynomial map $\gamma: C \rightarrow B$ with $\pi \circ \gamma$ equal to the identity on $C$. We will see that the identity component of a reductive abelian proaffine algebraic group over $F$ need not be a direct factor, i.e., have an algebraic group complement. Finally, we will see that there is an 
extension $[A, B, C]_{\pi}$ of (connected) pro-affine algebraic groups over $F$ such that there exists an abstract homomorphism $\gamma: C \rightarrow B$ with $\pi \circ \gamma=i_{c}$, but there is no rational homomorphism, in fact no polynomial map, $\lambda: C \rightarrow B$ with $\pi \circ \gamma=i_{C}$.

We begin with a lemma.

LEMMA 3.3. Let $X$ be a torsion-free abelian group, and $K$ any field. Then the units of the group algebra $K[X]$ are precisely the elements of the form $k x$, where $k \in K^{*}$ and $x \in X$.

Proof. If $\sum k_{i} x_{i}$ is a unit in $K[X]$ with inverse $\sum l_{j} y_{j}$, we may replace $K[X]$ with its $K$-subalgebra $K\left[X^{\prime}\right]$ where $X^{\prime}$ is the subgroup of $X$ generated by the $x_{i}$ 's and the $y_{j}$ 's. So we may assume that $X$ is finitely generated, hence a free abelian group of finite rank.

Now we have $K[X]=K\left[x_{1}, x_{1}^{-1}, \cdots, x_{n}, x_{n}^{-1}\right]$ where the $x_{i}$ 's are algebraically independent over $K$. Let $u$ be a unit of $K[X]$ ane $v$ its inverse. We may write $u=a / s$ and $v=b / t$ where $a$ and $b$ are in $K\left[x_{1}, \cdots, x_{n}\right]$ and $s$ and $t$ are monomials in $x_{1}, \cdots, x_{n}$ (with nonnegative exponents). Then $a b=s t$ and it follows immediately from unique factorization in $K\left[x_{1}, \cdots, x_{n}\right]$ that $a$ is $a K$-multiple of a monomial in $x_{1}, \cdots, x_{n}$, which gives the required form of $u$.

Now we can prove the following proposition.

\section{Proposition 3.4. Let}

$$
\text { *: } \quad 1 \longrightarrow A \longrightarrow B \stackrel{\pi}{\longrightarrow} C \longrightarrow 1
$$

be a short exact sequence of rational homomorphisms of reductive abelian pro-affine algebraic groups over $F$ with $C$ connected. Suppose there exists a polynomial map $\sigma: C \rightarrow B$ such that $\pi \circ \sigma$ equals the identity map on $C$. Then there exists a rational homomorphism $\gamma: C \rightarrow B$ such that $\pi \circ \gamma$ equals the identity map on $C$.

Proof. Write $Z=\hat{A}, Y=\hat{B}$, and $X=\hat{G}$. Then * induces, via $\hat{\imath}$, an exact sequence

$\widehat{*}$ :

$$
1 \longleftarrow Z \longleftarrow Y \stackrel{\hat{\pi}}{\longleftarrow} X \longleftarrow 1
$$

and the Hopf algebra sequence associated to ${ }^{*}$ is the sequence

$$
1 \longleftarrow F[Z] \longleftarrow F[Y] \stackrel{\pi^{\prime}}{\longleftarrow} F[X] \longleftarrow 1
$$

induced by $\hat{*}$.

Let $\sigma^{\prime}: F[Y] \rightarrow F[X]$ be the $F$-algebra homomorphism induced 
by $\sigma$. For $y \in Y, \sigma^{\prime}(y)$ is a unit in $F[X]$. Since $C$ is connected, $X$ is torsion-free, and we have $\sigma^{\prime}(y)=\lambda(y) \hat{\gamma}(y)$ with $\lambda(y) \in F^{*}$ and $\hat{\gamma}(y) \in X$. Because $\sigma^{\prime}$ is an algebra homomorphism, we have

$$
\lambda\left(y_{1}, y_{2}\right) \hat{\gamma}\left(y_{1} y_{2}\right)=\sigma^{\prime}\left(y_{1} y_{2}\right)=\sigma^{\prime}\left(y_{1}\right) \sigma^{\prime}\left(y_{2}\right)=\lambda\left(y_{1}\right) \lambda\left(y_{2}\right) \hat{\gamma}\left(y_{1}\right) \hat{\gamma}\left(y_{2}\right) .
$$

This implies, in particular, that $\hat{\gamma}: Y \rightarrow X$ is a homomorphism. Because $\pi \circ \sigma=i_{c}$, we have $\sigma^{\prime} \circ \pi^{\prime}=i_{F[X]}$, so that $\hat{\gamma}(\hat{\pi}(x))=x$ for all $x \in X$. Now the rational homomorphism $\lambda: C \rightarrow B$ induced by $\hat{\gamma}$ will suffice, for we have $\pi \circ \gamma=i_{c}$.

Now if we find an extension

$$
0 \longrightarrow X \longrightarrow Y \longrightarrow Z \longrightarrow 0
$$

of torsion-free abelian groups which is not split, we will obtain, by the above proposition, an extension

$$
1 \longrightarrow \check{Z} \longrightarrow \check{Y} \longrightarrow \check{X} \longrightarrow 1
$$

of connected abelian reductive pro-affine algebraic groups over $F$ for which no rational cross-section $\check{X} \rightarrow \check{Y}$ exists. Such examples abound, and one is given by

$$
0 \longrightarrow \Sigma \longrightarrow \pi \longrightarrow \pi / \Sigma \longrightarrow 0
$$

where $\pi$ is the direct product of a countably infinite number of copies of the integers and $\Sigma$ is the corresponding direct sum, viewed as a subgroup of $\pi$ in the natural way. This extension is not split because, for example, the element $(1 !, 2 !, 3 !, \cdots)+\Sigma$ of $\pi / \Sigma$ is divisible by every positive integer and no nonzero element of $\pi$ has this property.

Next, if we find an abelian group $X$ without $p$-torsion whose torsion subgroup $X_{t}$ is not a direct summand, we will obtain a reductive abelian pro-affine algebraic group $\check{X}$ over $F$ whose identity component $\left(X / X_{t}\right)$ has no algebraic group complement. For this we may take $X$ to be the product $\prod_{q \neq p} Z / q Z$ of one copy of each cyclic group of order a prime $q$ not equal to $p$. Then $X_{t}$ is the corresponding sum $\sum_{q \neq p} Z / q Z$. Then $X_{t}$ is not a direct summand because $X / X_{t}$ is divisible while $X$ contains no nontrivial divisible subgroup.

The identity component $G_{1}$ of a reductive abelian pro-affine algebraic group $G$ over $F$ is certainly a direct factor if either $G_{1}$ is injective or $G / G_{1}$ projective in the category of reductive abelian pro-affine algebraic groups over $F$. An object $H$ of this category is injective (projective) if and only if $\hat{H}$ is projective (injective) in the category of abelian groups without $p$-torsion. It follows that 
the injectives $H$ are the products $\Pi_{S} F^{*}$ of copies of the multiplicative group of $F$ (ovər arbitrary indexing sets) while the projectives $H$ are the products $\left(\Pi_{S} \widetilde{F}^{*}\right) \times\left(\Pi_{\alpha} H_{\alpha}\right)$ where $\widetilde{F}^{*}$ is the universal covering group of $F^{*}$ (whose Hopf algebra is $F\left[x^{1 / n}: n \in Z\right]$ ) and each $H_{\alpha}$ is isomorphic to the additive group $\boldsymbol{Z}_{q}$ of $q$-adic integers for some prime $q \neq p$.

A consequence of the following lemma is that any extension $[A, B, C]_{\pi}$ of reductive abelian pro-affine algebraic groups over $F$ with $A$ connected is abstractly split. Hence any such extension which is not algebraically split provides our third counterexample.

LEMmA 3.5. Let $D_{\alpha}$ form an inverse system of divisible abelian groups with homomorphisms $\phi_{\alpha, \beta}: D_{\beta} \rightarrow D_{\alpha}$ when $\alpha \leqq \beta$. Suppose that for each $\alpha$ and each positive integer $m, D_{\alpha}$ contains only finitely many elements of order $m$. Then the inverse limit $D=\underset{\alpha}{\lim } D_{\alpha}$ is divisible.

Proof. Let $x=\left(x_{\alpha}\right) \in D$ and let $m$ be a positive integer. Say that each $D_{\alpha}$ has $n_{\alpha}$ elements of order $m$. For each $\alpha$, let $A_{\alpha}=$ $\left\{y_{\alpha} \in D_{\alpha} \mid y_{\alpha}^{m}=x_{\alpha}\right\}$. Then $A_{\alpha}$ has $n_{\alpha}$ elements. Put $B_{\alpha}=\bigcap_{\beta \geq \alpha} \phi_{\alpha, \beta}\left(A_{\beta}\right)$. We claim that each $B_{\alpha}$ is nonempty. Suppose some $B_{\alpha}=\phi$. There exist indices $\beta_{1}, \cdots, \beta_{n_{\alpha}} \geqq \alpha$ with $y_{i} \notin \phi_{\alpha, \beta_{i}}\left(A_{\beta_{i}}\right)$ where $y_{1}, \cdots, y_{n_{\alpha}}$ are the elements of $A_{\alpha}$. Choose $\beta \geqq \beta_{1}, \cdots, \beta_{n_{\alpha}}$. Then $\phi_{\alpha, \beta}\left(A_{\beta}\right)=\phi$, which is absurd. So $B_{\alpha} \neq \phi$.

Now, by the projective limit theorem of [7], $\underset{\alpha}{\lim } B_{\alpha}$ is nonempty. If $y \in \underset{\alpha}{\lim } B_{\alpha}$, then $y^{m}=x$.

Theorem 7 on page 18 of [9] asserts that if the torsion subgroup of an abelian group is of bounded order, i.e., if the orders of its elements are bounded, then it is a direct summand. An abelian group $X$ (without $p$-torsion) is of bounded order if and only if $\breve{X}$ is of bounded order. This gives the following proposition.

Proposition 3.6. Let $G$ be a reductive abelian pro-affine algebraic group over $F$ with identity component $G_{1}$. If $G / G_{1}$ is of bounded order, then $G_{1}$ has an algebraic group complement in $G$. In particular, this holds if $G$ is affine.

8. Simply connected groups. Throughout this chapter, $F$ will be a fixed algebraically closed field of characteristic 0 , and all proaffine algebraic groups and varieties are defined over $F$. We will exhibit the universal properties which simply connected groups possess, 
and then investigate the question of the existence of cross-sections.

Proposition 4.1. Let $A, B$, and $H$ be connected pro-affine algebraic groups with $H$ simply connected. Suppose $\pi: A \rightarrow B$ is a group covering and $\eta: H \rightarrow B$ is a rational homomorphism. Then there exists a unique rational homomorphism $\mu: H \rightarrow A$ such that $\pi \circ \mu=\eta$.

Proof. Let $\gamma$ and $\alpha$ denote the restrictions to the fiber product $H \times_{\eta, \pi} A$ of the projections of $H \times A$ to the first and second factor, respectively, so both $\gamma: H \times_{\eta, \pi} A \rightarrow H$ and $\alpha: H \times_{\eta, \pi} A \rightarrow A$ are rational homomorphisms. $\gamma$ is surjective because $\pi$ is surjective and the kernel of $\gamma$ is the subgroup $1 \times K$ of $H \times A$ where $K$ is the kernel of $\pi$. This group is pro-finite. Because $H$ is connected, the restriction $\delta$ of $\gamma$ to the identity component $G$ of $H \times_{\eta, \pi} A$ is surjective. The kernel of $\delta$ is $G \cap(1 \times K)$ which is pro-finite, so $\delta: G \rightarrow H$ is a covering. Since $H$ is simply connected, $\delta$ is an isomorphism, so we may put $\mu=\alpha \circ \delta^{-1}$. Then $\mu$ satisfies the requirements of the proposition.

THEOREM 4.2. Let $A, B$, and $H$ be connected pro-affine algebraic groups (over $F$ ) with $H$ simply connected. Suppose $\pi: A \rightarrow B$ is a group covering and $\eta: H \rightarrow B$ is a polynomial map. Then for each $h$ in $H$ and $a$ in $A$ with $\eta(h)=\pi(a)$, there is a unique polynomial map $\mu: H \rightarrow A$ such that $\pi \circ \mu=\eta$ and $\mu(h)=a$.

First we observe that it will suffice to show that there exists some polynomial map $\mu: H \rightarrow A$ such that $\pi \circ \mu=\eta$. If such a map $\mu$ is given and $h \in H$ and $a \in A$ satisfy $\eta(h)=\pi(a)$, define $\mu^{\prime}: H \rightarrow A$ by $\mu^{\prime}\left(h^{\prime}\right)=\mu\left(h^{\prime}\right) \mu(h)^{-1} a$ for $h^{\prime}$ in $H$. Then $\pi \circ \mu^{\prime}=\eta$ and $\mu^{\prime}(h)=a$. If $\pi \circ \mu=\pi \circ \mu^{\prime}=\eta$ and $\mu(h)=\mu^{\prime}(h)=a$, then the map $H \rightarrow A$ sending each $h^{\prime}$ in $H$ to $\mu\left(h^{\prime}\right) \mu^{\prime}\left(h^{\prime}\right)^{-1}$ is a polynomial map from $H$ to the kernel of $\pi$. But the image of a connected pro-affine algebraic group (or variety) under a polynomial map is easily seen to be connected (i.e., its polynomial algebra is an integral domain). Since $\mu(h) \mu^{\prime}(h)^{-1}=1$, this implies that $\mu=\mu^{\prime}$.

We will first prove the result in the affine case. The proof will proceed under slightly weaker assumptions to facilitate handling the general case.

Lemma 4.3. Let $H, A, B, \pi$, and $\eta$ be as in Theorem 4.2 and assume that $H, A$, and $B$ are all affine. Then there is a polynomial map $\mu: H \rightarrow A$ such that $\pi \circ \mu=\eta$.

Proof. To begin, we do not assume $H$ is simply connected, but 
merely connected. Let $C$ denote the set of elements $(h, a)$ of $H \times A$ for which $\eta(h)=\pi(\alpha)$ and let $\gamma$ and $\alpha$ denote the restrictions to $C$ of the canonical projections of $H \times A$ onto the first and second factor, respectively. Then, clearly, $C$ is an algebraic subset of $H \times A$ and $\gamma$ and $\alpha$ are polynomial maps. $\gamma$ is surjective because $\pi$ is, and we have $\pi \circ \alpha=\eta \circ \gamma$.

Let the kernel $K$ of $\pi$ operate on $H \times A$ by multiplication in the second factor (note that $K$ is central in $A$ and $C$ is stable under this operation). Let us write $k \cdot(h, a)=(h, k a)$ for $k$ in $K$ and $(h, a)$ in $H \times A$.

We will write $P(V)$ for the polynomial algebra of any affine (or pro-affine) algebraic variety $V$ and $\alpha^{\prime}$ for the map $P(U) \rightarrow P(V)$ induced by a polynomial map $\alpha: V \rightarrow U$.

Let $C_{1}, \cdots, C_{q}$ be the maximal irreducible closed subsets of $C$, so $C=\bigcup_{i=1}^{q} C_{i}$. For each $i$, the Zariski closure $\overline{\gamma\left(C_{i}\right)}$ of $\gamma\left(C_{i}\right)$ is an irreducible closed subset of $H$ and $H=\bigcup_{i=1}^{q} \overline{\gamma\left(C_{i}\right)}$. Since $H$ is connected, i.e., irreducible, we have $H=\overline{\gamma\left(C_{i}\right)}$ for some $i$, say $H=\overline{\gamma\left(C_{1}\right)}$.

For each $i$, let $\gamma_{i}$ and $\alpha_{i}$ be the restrictions to $C_{i}$ of $\gamma$ and $\alpha$, respectively. Then $\gamma_{1}^{\prime}: P(H) \rightarrow P\left(C_{1}\right)$ is injective because $H=\overline{\gamma_{1}\left(C_{1}\right)}$. $P(H) \otimes P(A)$ is generated over $F$ by $P(H)$ and $P(A)$, and it follows that $P\left(C_{1}\right)$ is generated over $\gamma_{1}^{\prime}(P(H))$ by $\alpha_{1}^{\prime}(P(A))$. Also each element of $\alpha_{1}^{\prime}(P(A))$ is integral over $\alpha_{1}^{\prime}\left(\pi^{\prime}(P(B))\right)=\gamma_{1}^{\prime}\left(\eta^{\prime}(P(B))\right) \subset \gamma_{1}^{\prime}(P(H))$. Thus $P\left(C_{1}\right)$ is integral over $\gamma_{1}^{\prime}(P(H))$. This shows that $\gamma_{1}$ is a finite dominant morphism in the sense of [9]. It follows from the proposition on page 31 of that book that $\gamma_{1}\left(C_{1}\right)=H$ and that $\gamma_{1}$ is a closed map.

Next we show that $P\left(C_{1}\right)$ is an unramified extension of $\gamma_{1}^{\prime}(P(H))$ (cf. [3]). For this, let $M$ be a $P\left(C_{1}\right)$-module and $\delta: P\left(C_{1}\right) \rightarrow M$ a derivation with $\delta \circ \gamma_{1}^{\prime}=0$. View $M$ as a $P(A)$-module via $\alpha_{1}^{\prime}$. Then $\delta \circ \alpha_{1}^{\prime}: P(A) \rightarrow M$ is a derivation and $\delta \circ \alpha_{1}^{\prime} \circ \pi^{\prime}=\delta \circ \gamma_{1}^{\prime} \circ \pi^{\prime}=0$. Because $P(A)$ is an unramified extension of $\pi^{\prime}(P(B))$, we have $\delta \circ \alpha_{1}^{\prime}=0$. So $\delta$ vanishes on $\gamma_{1}^{\prime}(P(H))$ and on $\alpha_{1}^{\prime}(P(A))$. These generate $P\left(C_{1}\right)$, so we have $\delta=0$ and thus $P\left(C_{1}\right)$ is an unramified extension of $\gamma_{1}^{\prime}(P(H))$. We now have that for each element of $C_{1}$ sent by $\gamma_{1}$ to the identity element of $H$, there is a unique structure of affine algebraic group on $C_{1}$ having that element as the identity and such that $\gamma_{1}$ is a group covering.

Finally, we assume that $H$ is simply connected, so that $\gamma_{1}$ is an isomorphism (choosing an identity element for $C_{1}$ ). We put $\mu=$ $\alpha_{1} \circ \gamma_{1}^{-1}$ and then $\mu$ satisfies the requirements of the lemma.

Continuing the discussion preceding the last paragraph of the proof, we observe that $K$ permutes the $C_{i}$ 's and since $\gamma_{1}$ is surjective it is clear that $C=\bigcup_{k \in K} k \cdot C_{1}$. So for each $i$, there is a $k_{i}$ in 
$K$ with $C_{i}=k_{i} \cdot C_{1}$ and $K$ permutes the $C_{i}$ 's transitively. Since $\gamma_{1}$ is a group covering, all the fibers of $\gamma_{1}$ (over the various points of $H$ ) have the same cardinality $n$. Since, for each $i, \gamma_{i}$ coincides with the map $\gamma_{1} \circ \delta_{i}$ where $\delta_{i}: C_{i} \rightarrow C_{1}$ sends each $c_{i}$ in $C_{i}$ to $k_{i}^{-1} \cdot c_{i}$, and $\delta_{i}$ is a variety isomorphism, all the fibers of $\gamma_{i}$ have cardinality $n$. It follows that the $C_{i}$ 's are mutually disjoint. Indeed, we have $n q=\sum_{i=1}^{q}\left|\gamma_{i}^{-1}(h)\right|$ for every $h$ in $H$. If the $C_{i}$ 's are not mutually disjoint, we have that $n q=\sum_{i=1}^{q}\left|\gamma_{i}^{-1}(h)\right|>\left|\gamma^{-1}(h)\right|=|K|$ for some $h$ in $H$. Then we have that $\sum_{i=1}^{q}\left|\gamma_{i}^{-1}(h)\right|>\left|\gamma^{-1}(h)\right|$ for all $h$ in $H$. From this we see that $\gamma\left(\bigcup_{i \neq j}\left(C_{i} \cap C_{j}\right)\right)=H$, contradicting that $\operatorname{dim}\left(\mathbf{U}_{i \neq j}\left(C_{i} \cap C_{j}\right)\right)<\operatorname{dim}(C)=\operatorname{dim}(H)$.

We see also now that for each $i$ and each element of $C_{i}$ sent by $\gamma_{i}$ to the identity element in $H$, there is a unique structure of affine algebraic group on $C_{i}$ having that element as the identity such that $\gamma_{i}$ is a group covering.

Proof of Theorem 4.2. We begin by choosing $a_{1}$ in $A$ with $\pi\left(a_{1}\right)=\eta(1)$. Given any finitely generated Hopf subalgebra $R$ of $P(A)$, let $S=\left(\pi^{\prime}\right)^{-1}(R)$ and let $T$ be the smallest Hopf subalgebra of $P(H)$ containing $\eta^{\prime}(S)$. Since $\pi^{\prime}$ is injective, we may view $S$ as the intersection of $R$ with the Hopf subalgebra $P(B)$ of $P(A)$. The proof of Proposition 3.1 of [3] shows that $R$ is an integral unramified extension of $S$, and a finitely generated $S$-module. So we may apply the well-known Artin-Tate lemma. (cf. Theorem 1.13 of [8]) to conclude that $S$ is finitely generated as a $F$-algebra. Since the smallest Hopf subalgebra of a Hopf algebra containing a given finitely generated subalgebra is finitely generated as an algebra, $T$ is also finitely generated. We have commutative diagrams
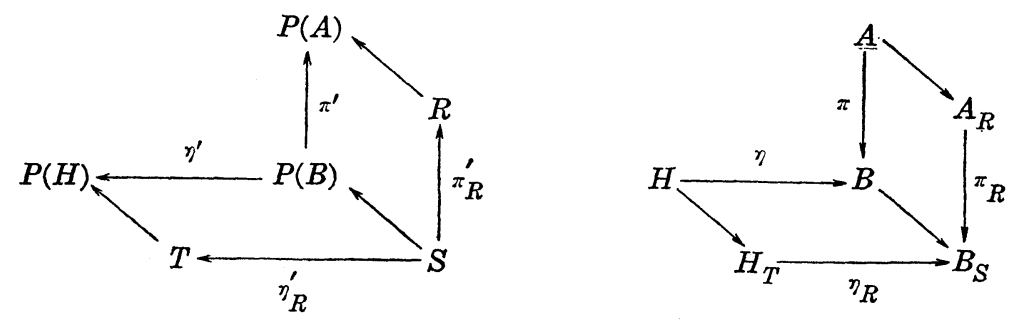

and $\pi_{R}$ is a covering of affine algebraic groups.

Let $\left(a_{1}\right)_{R}$ denote the image of $a_{1}$ in $A_{R}$. Define $C^{R} \subset H_{T} \times A_{R}$, $C_{i}^{R}, \gamma^{R}, \gamma_{i}^{R}, \alpha^{R}, \alpha_{i}^{R}$ as in the proof of Lemma 4.3, and fix notation so that $\left(1,\left(a_{1}\right)_{R}\right)$ belongs to $C_{1}^{R}$. We endow $C_{1}^{R}$ with the structure of affine algebraic group having $\left(1,\left(a_{1}\right)_{R}\right)$ as identity such that $\gamma_{1}^{R}$ is a covering. Because $H$ is simply connected, there is a unique (surjec- 
tive) rational homomorphism $\tau_{R}: H \rightarrow C_{1}^{R}$ making a commutative diagram

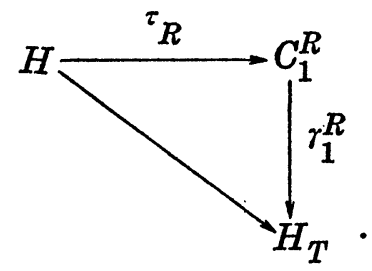

Define $\mu_{R}^{\prime}=\tau_{R}^{\prime} \circ\left(\alpha_{1}^{R}\right)^{\prime}: R \rightarrow P(H)$. Then we have

$$
\left.\left.\mu_{R}^{\prime} \circ \pi_{R}^{\prime}=\tau_{R}^{\prime} \circ\right) \alpha_{1}^{R}\right)^{\prime} \circ \pi_{R}^{\prime}=\tau_{R}^{\prime} \circ\left(\gamma_{1}^{R}\right)^{\prime} \circ \eta_{R}^{\prime}=\eta_{R}^{\prime} .
$$

Now suppose $R_{1}$ and $R_{2}$ are two finitely generated Hopf subalgebras of $P(A)$ with $R_{1} \subset R_{2}$. To make the notation more managable, we will use $i$, for $i=1,2$, as a subscript or superscript wherever analogy with the above would require using $R_{i}, S_{i}$ or $T_{i}$ as a subscript or superscript. We have a commutative diagram

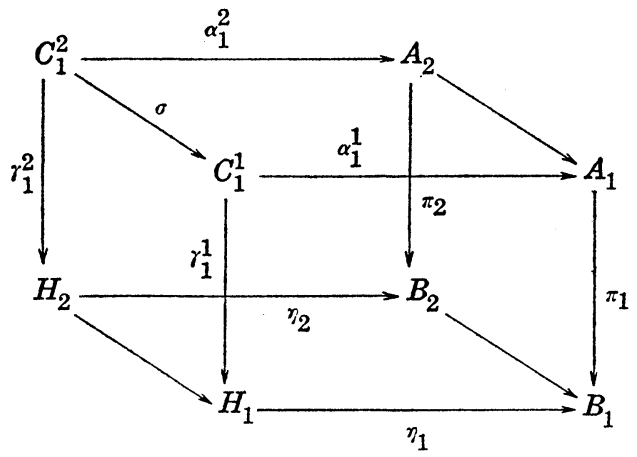

where $\sigma: C_{1}^{2} \rightarrow C_{1}^{1}$ is defined as follows: Let $\bar{\sigma}: H_{2} \times A_{2} \rightarrow H_{1} \times A_{1}$ be the canonical map. Then $\bar{\sigma}$ carries $C^{2}$ into $C^{1}$ and each component of the former into some component of the latter. Since $\bar{\sigma}\left(1,\left(a_{1}\right)_{2}\right)=$ $\left(1,\left(a_{1}\right)_{2}\right), \quad \bar{\sigma}$ induces a map $\sigma: C_{1}^{2} \rightarrow C_{1}^{1}$ making the above diagram commute.

We will now show that $\sigma$ is a homomorphism. Define $\theta: C_{1}^{2} \times$ $C_{1}^{2} \rightarrow C_{2}^{1}$ by $\theta(x, y)=\sigma(x) \sigma(y) \sigma(x y)^{-1}$. Then $\gamma_{1}^{1}(\theta(x, y))=1$ for every $x$ and $y$ in $C_{1}^{2}$, so $\theta$ is a polynomial map from $C_{1}^{2} \times C_{1}^{2}$ to the kernel of $\gamma_{1}^{1}$. Since the former is irreducible and the latter finite, $\theta$ is constant. Because $\sigma(1)=1$, we have $\theta(x, y)=1$ for every $x$ and $y$ in $C_{1}^{2}$, so $\sigma$ is a homomorphism. So the unicity of $\tau_{1}$ shows that the following diagram commutes. 


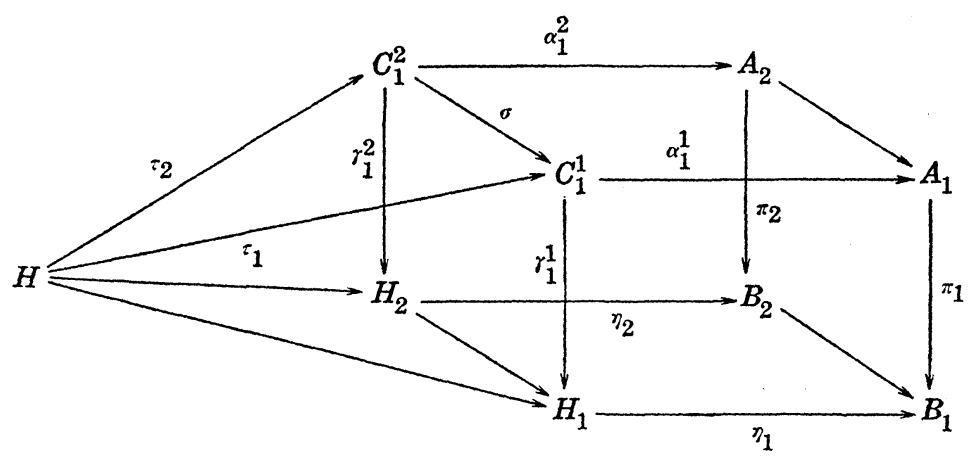

and so, in particular, does

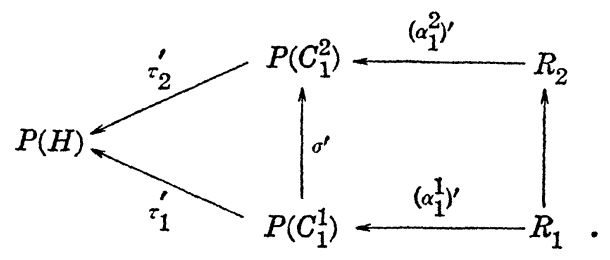

So the restriction of $\mu_{2}^{\prime}$ to $R_{1}$ coincides with $\mu_{1}^{\prime}$. Thus the $\mu_{R}^{\prime}$ 's together define an $F^{\prime}$-algebra homomorphism $\mu^{\prime}: P(A) \rightarrow P(H)$. Since $\mu_{R}^{\prime} \circ \pi_{R}^{\prime}=\eta_{R}^{\prime}$ for every $R$, we have $\mu^{\prime} \circ \pi^{\prime}=\eta^{\prime}$. Let $\mu: H \rightarrow A$ be the induced polynomial map. Then $\pi \circ \mu=\eta$, and the theorem is proved.

Now we turn to the question of the existence of cross-sections. Suppose $[K, G, H]_{\pi}$ is an extension of pro-affine algebraic groups (over $F$ ).

A cross-section is a polynomial map $\sigma: H \rightarrow G$ such that $\pi \circ \sigma$ is the identity map on $H$. We will show that if $H$ is simply connected a cross section-exists. We will obtain as a corollary the same result when $K$ is unipotent. So we assume $[K, G, H]_{\pi}$ is as above with $H$ simply connected. Let $K_{r}$ be a maximal reductive algebraic subgroup of $K$ and let $G_{r}$ be a maximal reductive algebraic subgroup of $G$ containing $K_{r}$. Then $H_{r}=\pi\left(G_{r}\right)$ is a maximal reductive algebraic subgroup of $H$ and we have two extensions of pro-affine algebraic group $\left[K_{u}, G_{u}, H_{u}\right]_{\pi_{u}}$ and $\left[K_{r}, G_{r}, H_{r}\right]_{\pi_{r}}$ where $\pi_{u}$ and $\pi_{r}$ are the obvious restrictions of $\pi$. Cross-sections for these two extensions give a cross-section for $[K, G, H]_{\pi}$ in the obvious manner. So we have reduced to the cases where all the groups are unipotent or all are reductive, and we still have that $H$ is simply connected in either of the two cases, by virtue of Theorem 5.2 of [3].

If all the groups are reductive, $G$ may not be connected but the identity component $G_{1}$ of $G$ is mapped onto $H$ by $\pi$ because $H$ is connected. So we may assume that $G$ is connected (then so is $K$ because $H$ is simply connected). Let $C_{1}(G), C_{1}(H), G^{\prime}$, and $H^{\prime}$ 
be as in Lemma 1.1, so that $H=C_{1}(H) \times H^{\prime}$. We have extensions $\left[K \cap C_{1}(G), G_{1}(G), C_{1}(H)\right]_{\pi}$ and $\left[K \cap G^{\prime}, G^{\prime}, H^{\prime}\right]_{\pi}$, where we use the symbol $\pi$ by abuse of notation. So in the case where all the groups are reductive we reduce further to the two cases where all are abelian or both $G$ and $H$ are semisimple, i.e., inverse limits of semisimple affine algebraic groups.

9. The reductive abelian case. Let $H$ be a simply connected reductive abelian pro-affine algebraic group over $F$. Then $\hat{H}$ is torsionfree and by considering the diagrams

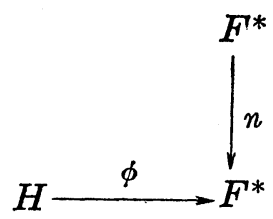

where $\phi \in \hat{H}$ and the positive integer $n$ denotes the $n$th power map, we see that $\hat{H}$ is divisible. Now if $[K, G, H]_{\pi}$ is an extension of reductive abelian pro-affine algebraic groups with $H$ simply connected, then $\hat{H}$ is divisible so it is a direct summand of $\hat{G}$ and the extension $[K, G, H]_{\pi}$ is split by a rational homomorphism. In particular, a cross-section exists.

10. The unipotent case. If $(G, A)$ is any pro-affine algebraic group (over $F$ as above), the Lie algebra $\mathscr{L}(G)$ of $G$ consists of all differentiations $\delta: A \rightarrow F$, i.e., $F$-linear maps $\delta$ for which $\delta(f g)=\delta(f) c(g)+$ $c(f) \delta(g)$ for all $f$ and $g$ in $A$ where $c: A \rightarrow F$ is the counit of $A$ (or the identity element of $G$ ). The bracket $[\delta, \varepsilon]$ of $\delta$ and $\varepsilon$ in $\mathscr{L}(G)$ is $(\delta \otimes \varepsilon-\varepsilon \otimes \delta) \circ \gamma$ where $\gamma$ is the comultiplication in $A$. For each finitely generated Hopf subalgebra $S$ of $A$, we have the restriction map $\mathscr{L}(G) \rightarrow \mathscr{L}\left(G_{S}\right)$, where $G_{S}=\mathscr{G}(S)$, and $\mathscr{L}(G)$ is clearly equal to the inverse limit of the $\mathscr{L}\left(G_{S}\right)$ 's. The projective limit theorem may be used to show that each restriction map $\mathscr{L}(G) \rightarrow \mathscr{L}\left(G_{S}\right)$ is surjective.

If $\tau: G \rightarrow H$ is a rational homomorphism, the differential $\tau: \mathscr{L}(G) \rightarrow$ $\mathscr{L}(H)$ is defined via the map $\tau^{\prime}: B \rightarrow A$ where $B$ is the polynomial algebra of $H$.

Suppose $(G, A)$ is a unipotent pro-affine algebraic group. Let $p_{S}: G \rightarrow G_{S}, p_{S, T}: G_{T} \rightarrow G_{S}, p_{S}^{0}: \mathscr{L}(G) \rightarrow \mathscr{L}\left(G_{S}\right)$ and $p_{S, T}^{0}: \mathscr{L}\left(G_{T}\right) \rightarrow \mathscr{L}\left(G_{S}\right)$ denote the obvious restriction maps when $S$ and $T$ are finitely generated Hopf subalgebras of $A$ with $S \subseteq T$. Then $p_{S}^{0}$ and $p_{S, T}^{0}$ are the differentials of $p_{S}$ and $p_{S, T}$, respectively. By Theorem 10.1 of [8] we have an isomorphism 


$$
\exp _{G_{S}}: \mathscr{L}\left(G_{S}\right) \longrightarrow G_{S}
$$

of affine algebraic varieties where the polynomial algebra of $\mathscr{L}\left(G_{S}\right)$ is generated as an $F$-algebra by the linear functions $\mathscr{L}\left(G_{S}\right) \rightarrow F$. By Theorem 10.2 of [8], we have $p_{S, T} \circ \exp _{G_{T}}=\exp _{G_{S}} \circ p_{S, T}^{0}$. If we give to $\mathscr{L}(G)$ the structure of pro-affine algebraic variety coming from the representation $\mathscr{L}(G)=\underset{\leftarrow}{\lim } \mathscr{L}\left(G_{S}\right)$, the above shows that we have an isomorphism

$$
\exp _{G}: \mathscr{L}(G) \longrightarrow G
$$

of pro-affine algebraic varieties satisfying $p_{S} \circ \exp _{G}=\exp _{G_{S}} \circ p_{S}^{0}$ for all $S$ as above. Let $\log _{G}$ denote the inverse of $\exp _{G}$. We see that $A$ is generated as an $F$-algebra by functions of the form $\lambda \circ p_{S}^{0} \circ \log _{G}$ where $S$ is as above and $\lambda: \mathscr{L}\left(G_{S}\right) \rightarrow F$ is a linear function.

Now let $[K, G, H]_{\pi}$ be an extension of unipotent pro-affine algebraic groups. We have a commutative diagram

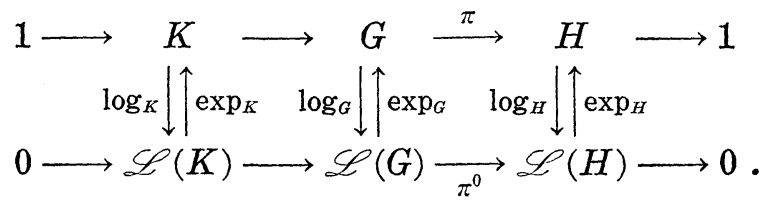

We will choose a linear map $\sigma^{0}: \mathscr{L}(H) \rightarrow \mathscr{L}(G)$ such that $\pi^{0} \circ \sigma^{0}$ is the identity map on $\mathscr{L}(H)$. Then we will put $\sigma=\exp _{G} \circ \sigma^{0} \circ \log _{H}$ and $\pi \circ \sigma$ will coincide with the identity map on $H$. However, some care must be taken to insure that $\sigma^{0}$, hence also $\sigma$, is a polynomial map. Let $A$ and $B$ denote the polynomial algebras of $G$ and $H$, respectively. Then $A$ is generated by elements of the form $\lambda \circ p_{S}^{0} \circ \log _{G}$ where $S$ is a finitely generated Hopf subalgebra of $A$ and $\lambda: \mathscr{L}\left(G_{S}\right) \rightarrow F$ a linear function. If, for each such function, there is a finitely generated Hopf subalgebra $T$ of $B$ and a linear function $\mu: \mathscr{L}\left(H_{T}\right) \rightarrow F$ such that $\lambda \circ p_{S}^{0} \circ \sigma^{0}=\mu \circ q_{T}^{0}$, where $q_{T}^{0}: \mathscr{L}(H) \rightarrow \mathscr{L}\left(H_{T}\right)$ is the canonical map, then it will follow that $\sigma$ is a polynomial map.

If $L$ is an inverse limit of finite dimensional vector spaces $L_{S}$, denote by $L^{\#}$ the space of all linear functions $L \rightarrow F$ which factor through one of the canonical maps $L \rightarrow L_{S}$. $L^{\sharp}$ may be viewed as the direct limit of the spaces dual to the $L_{S}$ 's. Let $\left(L^{\sharp}\right)^{0}$ denote the full dual of $L^{\sharp}$. It is easily seen that the natural map $L \rightarrow\left(L^{\sharp}\right)^{0}$ sending each element of $L$ to the evaluation at that element is a linear isomorphism.

In our situation, it is clear that $\mathscr{L}(H)^{\#} \circ \pi^{0}$ is contained in $\mathscr{L}(G)^{\sharp}$, so $\pi^{0}$ induces an injective linear map $\pi^{\sharp}: \mathscr{L}(H)^{\sharp} \rightarrow \mathscr{L}(G)^{\sharp}$. Let $\sigma^{\sharp}: \mathscr{L}(G)^{\sharp} \rightarrow \mathscr{L}(H)^{\sharp}$ be a linear map such that $\sigma^{\sharp} \circ \pi^{\sharp}$ equals the identity map on $\mathscr{L}(H)^{\sharp}$. Let $\sigma^{0}: \mathscr{L}(H) \rightarrow \mathscr{L}(G)$ be the map induced 
by $\sigma^{\sharp}$ via the identifications $\mathscr{L}(H)=\left(\mathscr{L}(H)^{\sharp}\right)^{0}$ and $\mathscr{L}(G)=\left(\mathscr{L}(G)^{\sharp}\right)^{0}$, so $\pi^{0} \circ \sigma^{0}=i_{\mathscr{L}(H)}$. Then we have that $\mathscr{L}(G)^{\#} \circ \sigma^{0}$ is contained in $\mathscr{L}(H)^{\sharp}$, which is precisely the condition cited above which shows that $\sigma=\exp _{G} \circ \sigma^{0} \circ \log _{H}$ is a polynomial map, and $\pi \circ \sigma=i_{H}$.

The semisimple case. Let $[K, G, H]_{\pi}$ be an extension in which $G$ and $H$ are connected and semisimple and $H$ is simply connected. We reduce further to the case where $G$ is also simply connected. Let $p: G^{\prime} \rightarrow G$ be a universal covering of $G$ and let $K^{\prime}$ be the kernel of $\pi \circ p$. Then we have an extension $\left[K^{\prime}, G^{\prime}, H\right]_{\pi \circ p}$ where $G^{\prime}$ and $H$ are both semisimple and simply connected. Following a cross-section for this extension by the map $p$ gives a cross-section for $[K, G, H]_{\pi}$. So we may assume that $G$ is simply connected. We will show that $G$ has a unique normal algebraic subgroup such that the restriction of $\pi$ to this subgroup is an isomorphism.

Let $A$ and $B$ denote the polynomial algebras of $G$ and $H$, respectively. If $S$ is a finitely generated Hopf subalgebra of $A$, let $q_{S}: G_{S}^{\prime} \rightarrow G_{S}$ be the universal covering of $G_{S}$ given in $\S 3$ of [4]. Then $G_{S}^{\prime}$ is semisimple affine algebraic group whose polynomial algebra we denote by $S^{\prime}$. Since $G$ is simply connected, there is a unique rational homomorphism $r: G \rightarrow G_{S}^{\prime}$ such that $q_{S} \circ r=p_{S}$, where $p_{S}: G \rightarrow G_{S}$ is the restriction homomorphism. It follows that $r$ is surjective, so we may view $S^{\prime}$ as a Hopf subalgebra of $A$. Thus $A$ is equal to the union of its finitely generated Hopf subalgebras $S$ with the property that $\left(G_{S}, S\right)$ is simply connected. The same is true of $B$.

Suppose $S \subset A$ as above and that $\left(G_{S}, S\right)$ is simply connected. View the map $\pi^{\prime}: B \rightarrow A$ as an identification, i.e., view $B$ as a Hopf subalgebra of $A$, and let $T=B \cap S$. Then $T$ is finitely generated and we have a commutative diagram

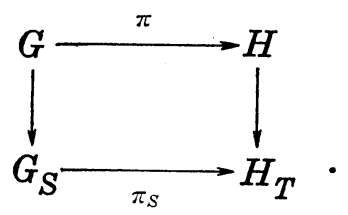

We wish to show that $H_{T}$ is simply connected. Suppose $H_{T}^{\prime} \rightarrow H_{T}$ is a covering (which implies that $H_{T}^{\prime}$ is an affine algebraic group). Because $G_{S}$ is simply connected, we get a commutative diagram

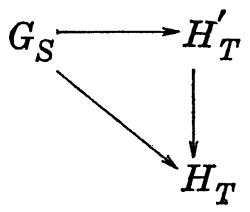


and the map $G_{S} \rightarrow H_{T}^{\prime}$ is surjective. This gives a commutative diagram

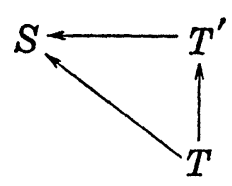

by which we may view the polynomial algebra $T^{\prime}$ of $H_{T}^{\prime}$ as an integral unramified extension of $T$ in $S$. We must show that $T^{\prime}=T$. We will show that $T^{\prime} B$ is a profinite extension of $B$ in $A$. If $V$ is a finitely generated Hopf subalgebra of $B$ containing $T$, then $T^{\prime} V$ is clearly an integral extension of $V$. There is a natural surjection $D_{T}\left(T^{\prime}\right) \otimes_{T} V \rightarrow D_{V}\left(T^{\prime} V\right)$ for which $\left(t_{1}^{\prime} \otimes t_{2}^{\prime}+J_{1}\right) \otimes v$ is sent to $t_{1}^{\prime} \otimes$ $t_{2}^{\prime} v=J_{2}$ where $D_{T}\left(T^{\prime}\right)=T^{\prime} \otimes_{T} T^{\prime} / J_{1}$ and $D_{V}\left(T^{\prime} V\right)=T^{\prime} V \bigotimes_{V} T^{\prime} V / J_{2}$ are the spaces of Kaehler differentials. Since $D_{T}\left(T^{\prime}\right)=(0)$, we also have $D_{V}\left(T^{\prime} V\right)=(0)$. So $T^{\prime} V$ is an integral unramified extension of $V$. By Theorem 4.3 of [3], this shows that $T^{\prime} B$ is a profinite extension of $B$. Now, because $(H, B)$ is simply connected, we have $T^{\prime} B=B$, i.e., $T^{\prime} \subset B$. Thus $T^{\prime} \subset B \cap S=T$, so $T^{\prime}=T$ and $H_{T}$ is simply connected.

Let $S$ and $T$ be as above. Then $\mathscr{L}\left(G_{S}\right)$ is a finite dimensional semisimple Lie algebra over $F$, so it has a unique ideal with the property that the restriction of $\pi_{S}^{0}: \mathscr{L}\left(G_{S}\right) \rightarrow \mathscr{L}\left(H_{T}\right)$ to this ideal is an isomorphism. This ideal is semisimple, hence algebraic (cf. Corollary 13.4 of [8]). Because $H_{T}$ is simply connected, there is a unique rational homomorphism $\sigma_{S}: H_{T} \rightarrow G_{S}$ whose differential $\sigma_{S}^{0}$ coincides with the inverse of the above isomorphism by Theorem 3.1 of [4]. Then $\sigma_{S}\left(H_{T}\right)$ is the unique normal algebraic subgroup of $G_{S}$ such that the restriction of $\pi_{S}$ to $\sigma_{S}\left(H_{T}\right)$ is an isomorphism. In view of the unicity, it is clear that $\lim _{S} \sigma_{S}\left(H_{T}\right)$ is the unique normal algebraic subgroup of $G$ such that the restriction of $\pi$ to this subgroup is an isomorphism, where $S$ ranges over the finitely generated Hopf subalgebras of $A$ for which $\left(G_{S}, S\right)$ is simply connected. This completes the proof of the following theorem.

THEOREM 4.4. Let $[K, G, H]_{\pi}$ be an extension of pro-affine algebraic groups over an algebraically closed field of characteristic 0 and suppose that $H$ is simply connected. Then there exists a crosssection, i.e., a polynomial map $\sigma: H \rightarrow G$ such that $\pi \circ \sigma$ equals the identity map on $H$.

We saw that in the reductive abelian case, the extension was split. After reducing the semisimple case to the case where $G$ was 
also simply connected, we saw that in that case too the extension was split. The nature of that latter reduction makes it clear that the same is true without it.

If $[K, G, H]_{\pi}$ is an extension where $H$ is reductive and simply connected, we may choose $K_{r}$ and $G_{r}$ as above with $K_{r} \subseteq G_{r}$ and then $\pi\left(G_{r}\right)=H$. Then, replacing $G_{r}$ with its identity component and $K_{r}$ with its identity component, we get extensions $\left[K_{r} \cap C_{1}\left(G_{r}\right), C_{1}\left(G_{r}\right)\right.$, $\left.C_{1}(H)\right]_{\pi}$ and $\left[K_{r} \cap G_{r}^{\prime}, G_{r}^{\prime}, H\right]_{\pi}$ as above and both are split. Since $C_{1}\left(G_{r}\right)$ is central in $G_{r}$, the extension $\left[K_{r}, G_{r}, H\right]_{\pi}$ is split. Hence so is $[K, G, H]_{\pi}$. So our proof of Theorem 4.4 also proved the following theorem.

THEOREM 4.5. Let $[K, G, H]_{\pi}$ be an extension of pro-affine algebraic groups over an algebraically closed field of characteristic 0 and suppose that $H$ is reductive and simply connected. Then the extension is split.

Next suppose that $[K, G, H]_{\pi}$ is an extension in which $K$ is unipotent. Then $K$ is contained in the unipotent radical $G_{u}$ of $G$. By the above theorem, since $H_{u}$ is simply connected, there is a crosssection for the extension $\left[K, G_{u}, H_{u}\right]_{\pi_{u}}$. If $G_{r}$ is a maximal reductive algebraic subgroup of $G$, then the restriction of $\pi$ to $G_{r}$ is an isomorphism onto a maximal reductive algebraic subgroup of $H$. The inverse of this isomorphism together with the cross-section for $\left[K, G_{u}, H_{u}\right]_{\pi_{u}}$ gives a cross-section for $[K, G, H]_{\pi}$. This proves the following corollary.

COROLLARY 4.6. Let $[K, G, H]_{\pi}$ be an extension of pro-affine algebraic groups over an algebraically closed field of characteristic 0 and suppose that $K$ is unipotent. Then there exists a crosssection.

The existence of cross-sections makes possible a description of group extensions in terms of rational cohomology. This is most practical in the case of an abelian kernel. Let $A$ and $H$ be proaffine algebraic groups (over $F$ ) with $A$ abelian and let $\eta: H \rightarrow$ Aut $(A)$ be a homomorphism making $A$ an $H$-group. We denote by $C^{n}(H, A)$ the set of all rational $n$-cochains for $H$ in $A$, i.e., the set of all polynomial maps $H \times \cdots \times H$ ( $n$ copies) $\rightarrow A$, for each positive integer $n$ and we put $C^{0}(H, A)=A$. Then we have the usual boundary operator $\delta: C^{n}(H, A) \rightarrow C^{n+1}(H, A)$ whose kernel we denote by $Z^{n}(H, A)$ and whose image we denote by $B^{n+1}(H, A)$. We have $B^{n}(H, A) \subset Z^{n}(H, A)$ for $n \geqq 1$, as usual. Each $B^{n}(H, A), Z^{n}(H, A)$, and $C^{n}(H, A)$ is an abelian group under valuewise multiplication. 
Just as with abstract groups, we have an injective homomorphism

$$
Z^{2}(H, A) / B^{2}(H, A) \longrightarrow \operatorname{Ext}(H, A, \eta) \text {. }
$$

The image of this homomorphism consists of the classes of extensions of $A$ by $H$ inducing $\eta$ for which there exists a cross-section. So if $H$ is simply connected or $A$ is unipotent (i.e., an inverse limit of finite dimensional vector groups) the above map is an isomorphism.

If, in the above situation, $A$ is a finite dimentional vector group, we have a natural identification

$$
C^{n}(H, A)=A \otimes P\left(H^{n}\right)
$$

where $P\left(H^{n}\right)=P(H) \otimes \cdots \otimes P(H)$ ( $n$ copies $)$.

11. Reductions of Ext. We will use the tools we have accumulated to make several reductions for the problem of computing Ext in the category of pro-affine algebraic groups over an algebraically closed field $F$ of characteristic 0 . Modulo the question of the existence of extensions, Proposition 2.1 reduces our problem to the case of an abelian kernel. If $A$ is an abelian pro-affine algebraic group over $F$, the unipotent radical $A_{u}$ and maximal reductive algebraic subgroup $A_{r}$ of $A$ are both stable under all rational automorphisms of $A$. So by Proposition 2.5 we reduce to the cases in which the kernel is abelian and reductive or abelian and unipotent.

With Proposition 2.3 of [5], it is easy to see that if $A$ is a reductive abelian pro-affine algebraic group over $F$, then any algebraic subgroup of Aut $(A)$ is totally disconnected.

Suppose that $A$ and $H$ are pro-affine algebraic groups over $F$ with $A$ abelian and reductive, and that $\eta: H \rightarrow \operatorname{Aut}(A)$ makes $A$ into an $H$-group. Since the unipotent radical $H_{u}$ of $H$ is connected, $H_{u}$ operates trivially on $A$. Thus $H^{1}\left(H_{u}, A\right)=\operatorname{Hom}\left(H_{u}, A\right)=(0)$ and $A^{H_{u}}=A$. Also, an easy application of the structure theory with respect to unipotent and reductive subgroups shows that $\operatorname{Ext}\left(H_{u}, A\right)=$ (0), so $\operatorname{Ext}_{H_{u}}(H, A)=\operatorname{Ext}(H, A)$ (we suppress $\eta$ in the notation). So Proposition 2.2.2 shows that the inflation homomorphism is an isomorphism $\operatorname{Ext}\left(H / H_{u}, A\right) \rightarrow \operatorname{Ext}(H, A)$.

If we choose a maximal reductive algebraic subgroup $H_{r}$ of $H$, we obtain a commutative diagram of isomorphisms

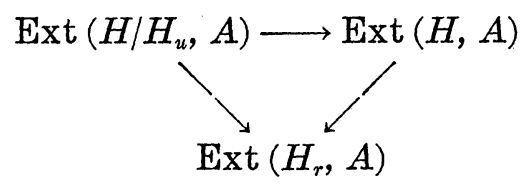

where each map is a lifting homomorphism associated with one of 
the three natural maps $H \rightarrow H / H_{u}, H_{r} \rightarrow H$, and $H_{r} \rightarrow H / H_{u}$.

(We have not spelled out the definition of a lifting homomorphism, but it is just like the inflation homomorphism. If $A$ is an abelian $H$-group and $\sigma: G \rightarrow H$ a rational homomorphism, the lifting homomorphism sends the class of $[A, E, H]_{\pi}$ to that of $\left[A, E \times_{\pi, \sigma} G, G\right]_{\pi}$, where $\pi^{\prime}$ is the restriction to $E \times_{\pi, \sigma} G$ of the projection of $E \times G$ onto the second factor. If $\sigma$ is the inclusion of an algebraic subgroup $G$ of $H$, then $E \times_{\pi, \sigma} G$ may be identified with $\pi^{-1}(G)$.)

Now let $H$ and $U$ be pro-affine algebraic groups over $F$ with $U$ unipotent and let $\eta: H \rightarrow O(U)$ be a homomorphism. Let $C$ denote the center of $U$ and $\eta_{0}: H \rightarrow \operatorname{Aut}(C)$ the map induced by $\eta$. Suppose that $\operatorname{Ext}(H, U, \eta)$ is nonempty, which implies that $\eta_{0}$ makes $C$ into an $H$-group.

Because $\operatorname{Ext}(H, U)$ is nonempty, by choosing a cross-section for some extension of $U$ by $H$ inducing $\eta$ we obtain a polynomial map $\eta^{\prime}: H \rightarrow \operatorname{Aut}(U)$ of $H$ into an algebraic subgroup of Aut $(U)$ such that $\eta^{\prime}$ followed by the canonical map Aut $(U) \rightarrow O(U)$ coincides with $\eta$. We may arrange that $\eta^{\prime}(1)$ is the identity automorphism on $U$.

If $x \in H$ and $\xi=\left[U, E, H_{u}\right]_{\alpha, \pi}$ is an extension inducing the restriction $\eta_{u}$ of $\eta$ to $H_{u}$, let $x \cdot \xi$ denote the extension $\left[U, E, H_{u}\right]_{\alpha_{x}, \pi_{x}}$ where $\alpha_{x}=\alpha \circ \eta^{\prime}(x)^{-1}$ and $\pi_{x}(e)=x \pi(e) x^{-1}$ for $e$ in $E$. One can easily verify that $x \cdot \xi$ induces $\eta_{u}$ for each $x$ in $H$ and that $1 \cdot \xi=\xi$. For $x$ and $y$ in $H$, the map $\eta^{\prime}(x) \circ \eta^{\prime}(y) \circ \eta^{\prime}(x y)^{-1}$ is an inner automorphism of $U$, say $\eta^{\prime}(x) \circ \eta^{\prime}(y) \circ \eta^{\prime}(x y)^{-1}=C_{\bar{u}}$ where $C_{\bar{u}}$ is the conjugation by the element $\bar{u}$ of $U$. If we use $C_{\bar{u}}$ also to denote the conjugation on $E$ by the element $\alpha(\bar{u})$ of $E$, then one can easily verify that $C_{\bar{u}}$ gives an equivalence of extensions $(x y) \cdot \xi \rightarrow x \cdot(y \cdot \xi)$. So we have an action of $H$ on $\operatorname{Ext}\left(H_{u}, U\right)$ such that each $x$ in $H$ sends the class of each $\xi$ as above to that of $x \cdot \xi$. We leave to the reader to verify that this action does not depend on the choice of the polynomial map $\eta^{\prime}$ lifting $\eta$ as above.

If $x$ belongs to $H_{u}$, we may choose $e$ in $E$ such that $\pi(e)=x$ and $e \alpha(u) e^{-1}=\alpha\left(\eta^{\prime}(x)\{u\}\right)$ for each $u$ in $U$. Then the map $E \rightarrow E$ sending each $e_{1}$ in $E$ to $e e_{1} e^{-1}$ gives an equivalence of extensions $x \cdot \xi \rightarrow \xi$. So the above action factors through $H / H_{u}$.

Suppose that $\left[U, E^{\prime}, H\right]_{\alpha^{\prime}, \pi^{\prime}}$ is an extension inducing $\eta$. The lifting map $\operatorname{Ext}(H, U) \rightarrow \operatorname{Ext}\left(H_{u}, U\right)$ sends its class to that of $\xi=$ [U, $\left.E, H_{u}\right]_{\alpha, \pi}$ where $E=\pi^{-1}\left(H_{u}\right)$ and $\alpha$ and $\pi$ are the obvious maps. For $x$ in $H$, we may choose $e^{\prime}$ in $E^{\prime}$ such that $\pi^{\prime}\left(e^{\prime}\right)=x$ and $e^{\prime} \alpha^{\prime}(u)\left(e^{\prime}\right)^{-1}=\alpha^{\prime}\left(\eta^{\prime}(x)\{u\}\right)$ for each $u$ in $U$. Then the map $E \rightarrow E$ sending each $e$ in $E$ to $e^{\prime} e\left(e^{\prime}\right)^{-1}$ gives an equivalence of extensions $x \cdot \xi \rightarrow \xi$. So the image of $\operatorname{Ext}(H, U)$ under the lifting map is contained in the $H$-fixed part $\operatorname{Ext}\left(H_{u}, U\right)^{H}$ of $\operatorname{Ext}\left(H_{u}, U\right)$. 
We specialize first to the case where $U=A$ is abelian. The following two propositions will show that if we assume further that $A$ is affine, then the lifting homomorphism is an isomorphism

$$
\operatorname{Ext}(H, A, \eta) \longrightarrow \operatorname{Ext}\left(H_{u}, A, \eta_{u}\right)^{I I} .
$$

Proposition 5.1. In the above situation with $A$ a unipotent affine H-group, the image of the lifting homomorphism coincides with $\operatorname{Ext}\left(H_{u}, A, \eta_{u}\right)^{H}$.

Proof. We have a natural action of $H$ on each $C^{n}\left(H_{u}, A\right)=$ $A \otimes P\left(H_{u}^{n}\right)$ for which each $x$ in $H$ sends each $a \otimes \lambda_{1} \otimes \cdots \otimes \lambda_{n}$ to $\eta(x)\{a\} \otimes\left(x \cdot \lambda_{1}\right) \otimes \cdots \otimes\left(x \cdot \lambda_{n}\right)$ where $(x \cdot \lambda)(y)=\lambda\left(x^{-1} y x\right)$ for $\lambda$ in $P\left(H_{u}\right)$ and $y$ in $H_{u}$. This action of $H$ on $C^{n}\left(H_{n}, A\right)$ stabilizes $Z^{n}\left(H_{u}, A\right)$ and $B^{n}\left(H_{u}, A\right)$. It is easy to verify that under the identification $\operatorname{Ext}\left(H_{u}, A\right)=Z^{2}\left(H_{u}, A\right) / B^{2}\left(H_{u}, A\right)$, the above two actions by $H$ coincide. Each $C^{n}\left(H_{u}, A\right)$ is a rational representation module for $H$, i.e., a sum of finite dimensional rational representation modules in the usual sense.

Let $H_{r}$ be a maximal reductive algebraic subgroup of $H$. Because $H_{r}$ is reductive, it follows that all of its rational representations are semisimple. So there is an $H_{r}$-stable subspace $S$ of $Z^{2}\left(H_{u}, A\right)$ such that $Z^{2}\left(H_{u}, A\right)=B^{2}\left(H_{u}, A\right) \oplus S$.

Now suppose that $\xi=\left[A, E, H_{u}\right]_{\alpha, \pi}$ is an extension inducing $\eta_{u}$ whose class is $H$-fixed. This class determines a unique element $f$ of $S$ and, because the class in $H$-fixed, we have $x \cdot f=f$ for every $x$ in $H_{r}$. Via $f$ we may identify $E$ with $A \times H_{u}$ endowed with the group structure given by $\left(a_{1}, y_{1}\right)\left(a_{2}, y_{2}\right)=\left(a_{1} \eta\left(y_{1}\right)\left\{a_{2}\right\} f\left(y_{1}, y_{2}\right), y_{1} y_{2}\right)$. Then $\alpha$ sends each $a$ in $A$ to $\left(a f(1,1)^{-1}, 1\right)$ and $\pi$ projects onto the second factor. Put $E^{\prime}=A \times H_{u} \times H_{r}$ and define a composition in $E^{\prime}$ by

$$
\left(a_{1}, y_{1}, x_{1}\right)\left(a_{2}, y_{2}, x_{2}\right)=\left(a_{1} \eta\left(y_{1} x_{1}\right)\left\{a_{2}\right\} f\left(y_{1}, x_{1} y_{2} x_{1}^{-1}\right), y_{1}\left(x_{1} y_{2} x_{1}^{-1}\right), x_{1} x_{2}\right) .
$$

One may verify that this makes $E^{\prime}$ a pro-affine algebraic group over $F$. The identity element is $\left(f(1,1)^{-1}, 1,1\right)$ and the inverse of $(a, y, x)$ is $\left(\eta\left(x^{-1} y^{-1}\right)\left\{\alpha f(1,1) f\left(y, y^{-1}\right)\right\}^{-1}, x^{-1} y^{-1} x, x^{-1}\right)$. Define $\alpha^{\prime}: A \rightarrow E^{\prime}$ and $\pi^{\prime}: E^{\prime} \rightarrow H$ by $\alpha^{\prime}(a)=\left(\alpha f(1,1)^{-1}, 1,1\right)$ and $\pi^{\prime}(a, y, x)=y x$ for $a$ in $A$, $y$ in $H_{u}$, and $x$ in $H_{r}$. Then both are rational homomorphisms and, in fact, $\xi^{\prime}=\left[A, E^{\prime}, H\right]_{\alpha^{\prime}, \pi^{\prime}}$ is an extension inducing $\eta$. The verifications are straightforward. One now easily sees that the class of $\xi^{\prime}$ is sent by the lifting homomorphism to that of $\xi$. This completes the proof.

This argument extends easily to the case where $A$ is a product 
of finite dimensional $H$-groups. If $A$ is any unipotent abelian proaffine $H$-group and if $H_{u}$ operates trivially on $A$, then one can show that $A$ is $H$-isomorphic with a product of finite dimensional $H$-groups so that we obtain the same result. But for now we must leave open the question of whether this extends to an arbitrary unipotent abelian pro-affine $H$-group.

Proposition 5.2. Under the same assumptions as in Proposition 5.1, the lifting homomorphism is injective.

\section{Proof. By Proposition 2.2.2 we have an exact sequence}

$$
\operatorname{Ext}\left(H / H_{u}, A^{H_{u}}\right) \longrightarrow \operatorname{Ext}_{H_{u}}(H, A) \longrightarrow H^{1}\left(H / H_{u}, H^{1}\left(H_{u}, A\right)\right)
$$

where $\operatorname{Ext}_{H_{u}}(H, A)$ is the kernel of the lifting homomorphism. We have that $\operatorname{Ext}\left(H / H_{u}, A^{H_{u}}\right)=(0)$ because $H / H_{u}$ is reductive and $A^{H_{u}}$ is unipotent. So it will suffice to show that the image of $\operatorname{Ext}_{H_{u}}(H, A)$ in $H^{1}\left(H / H_{u}, H^{1}\left(H_{u}, A\right)\right)$ is trivial.

The action of $H / H_{u}$ on $H^{1}\left(H_{u}, A\right)$ comes as follows: For any $x$ in $H$ and any 1-cochain $f: H_{u} \rightarrow A$ we define $x \cdot f: H_{u} \rightarrow A$ by $(x \cdot f)(y)=$ $\eta(x)\left\{f\left(x^{-1} y x\right)\right\}$. This gives an action of $H$ on $C^{1}\left(H_{u}, A\right)$ which stabilizes $Z^{1}\left(H_{u}, A\right)$ and $B^{1}\left(H_{u}, A\right)$, so induces an action of $H$ on $H^{1}\left(H_{u}, A\right)=$ $Z^{1}\left(H_{u}, A\right) / B^{1}\left(H_{u}, A\right)$ which factors through $H / H_{u}$. With the identification $C^{1}\left(H_{u}, A\right)=A \otimes P\left(H_{u}\right)$, this corresponds to the usual action described in the proof of Proposition 5.1. This shows that $H^{1}\left(H_{u}, A\right)$ is a rational $H / H_{u}$-module.

We call a rational 1-cocycle $d: H / H_{u} \rightarrow H^{1}\left(H_{u}, A\right)$ admissible if its image lies in a finite dimensional subspace of $H^{1}\left(H_{u}, A\right)$. If $d$ is a 1-coboundary, then it is clearly admissible. Let $Z_{*}^{1}\left(H / H_{u}, H^{1}\left(H_{u}, A\right)\right)$ denote the group of admissible 1-cocycles and put $H_{*}^{1}\left(H / H_{u}, H^{1}\left(H_{u}, A\right)\right)=$ $Z_{*}^{1}\left(H / H_{u}, H^{1}\left(H_{u}, A\right)\right) / B^{1}\left(H / H_{u}, H^{1}\left(H_{u}, A\right)\right)$. It is well-known that for any reductive affine (and hence pro-affine) algebraic group over $F$ and any finite dimensional rational representation module for that group, $H^{1}$ of that group in that module is trivial. Our $H_{*}^{1}$ is defined so as to make that result easily extend. So we have $H_{*}^{1}\left(H / H_{u}, H^{1}\left(H_{u}, A\right)\right)=(0)$.

We will now show that the image of $\operatorname{Ext}_{H_{u}}(H, A)$ is contained in $H_{*}^{1}\left(H / H_{u}, H^{1}\left(H_{u}, A\right)\right)$ which will complete the proof. Any extension of $A$ by $H$ inducing $\eta$ which is $H_{u}$-split may be represented by a 2-cocycle $f \in Z^{2}(H, A)$ which satisfies $f(y, z)=1$ for $y$ and $z$ in $H_{u}$. Given such an $f$, for $x$ in $H$ define $g(x): H_{u} \rightarrow A$ by $g(x)(y)=$ $f\left(x, x^{-1} y x\right) f(y, x)^{-1}$ for $y$ in $H_{u}$. One can verify that $g(x)$ is a 1cocycle $H_{u} \rightarrow A$ and that for $y$ in $H_{u}, g(y x)$ and $g(x)$ differ by a 1coboundary $H_{u} \rightarrow A$. So $g$ defines a map $H / H_{u} \rightarrow H^{1}\left(H_{u}, A\right)$ which may be shown to be a 1-cocycle. Tracing through the definition of 
the map $\operatorname{Ext}_{H_{u}}(H, A) \rightarrow H^{1}\left(H / H_{u}, H^{1}\left(H_{u}, A\right)\right)$, one can verify that the class of the extension represented by $f$ is sent to the class of this 1-cocycle. The map $(x, y) \mapsto g(x)(y)$ is given by an element of $A \otimes$ $P\left(H_{u}\right) \otimes P(H)$ and it is clear that as $x$ ranges over $H$, the maps $g(x)$ span a finite dimensional space of functions $H_{u} \rightarrow A$. This shows that the image of $\operatorname{Ext}_{H_{u}}(H, A)$ is contained in $H_{*}^{1}\left(H / H_{u}, H^{1}\left(H_{u}, A\right)\right)$.

Now let $H$ and $U$ be pro-affine algebraic groups over $F$ with $U$ unipotent and let $\eta: H \rightarrow O(U)$ be a homomorphism such that $\operatorname{Ext}(H, U, \eta)$ is nonempty. This implies that the induced homomorphism $\eta_{0}: H \rightarrow$ Aut $(C)$, where $C$ is the center of $U$, makes $C$ into an $H$-group. We have faithful transitive actions of $\operatorname{Ext}(H, C)$ on $\operatorname{Ext}(H, U)$ and $\operatorname{Ext}\left(H_{u}, C\right)$ on $\operatorname{Ext}\left(H_{u}, U\right)$, where we omit the various maps induced by $\eta$ in the notation.

If $\lambda$ and $\mu$ are extensions representing elements of $\operatorname{Ext}\left(H_{u}, C\right)$ and $\operatorname{Ext}\left(H_{u}, U\right)$, respectively, then one can verify that $x \cdot(\lambda \boxplus \mu)=$ $x \cdot \lambda \boxplus x \cdot \mu$. It follows from the fact that the action of $\operatorname{Ext}\left(H_{u}, C\right)$ on $\operatorname{Ext}\left(H_{u}, U\right)$ is faithful and transitive that if the class of $\mu$ is $H$-fixed, then $\operatorname{Ext}\left(H_{u}, U\right)^{I I}$ is equal to the set of classes of $\lambda \boxplus \mu$ where $\lambda$ ranges over a set of representatives for the elements of $\operatorname{Ext}\left(H_{u}, C\right)^{H}$.

Now suppose that $C$ is affine. Then the lifting homomorphism $\operatorname{Ent}(H, C) \rightarrow \operatorname{Ext}\left(H_{u}, C\right)^{H}$ is an isomorphism. It follows immediately from Proposition 2.4 that the lifting map $\operatorname{Ext}(H, U) \rightarrow \operatorname{Ext}\left(H_{u}, U\right)^{H}$ is a bijection, so we have proved the following theorem.

THEOREM 5.3. In the notation introduced above, where $U$ is unipotent and the center $C$ of $U$ is affine, if $\operatorname{Ext}(H, U)$ is nonempty, then the lifting map $\operatorname{Ext}(H, U) \rightarrow \operatorname{Ext}\left(H_{u}, U\right)^{H}$ is a bijection. In particular, this holds if $U$ is a unipotent affine algebraic group over $F$ and $\operatorname{Ext}(H, U)$ is nonempty.

Let us return now to the reductive situation. If $A$ and $H$ are reductive pro-affine algebraic groups over $F$ and $A$ is an abelian $H$-group via the homomorphism $\eta: H \rightarrow \operatorname{Aut}(A)$, then the identity component $H_{1}$ of $H$ is contained in the kernel of $\eta$. The tools we have at hand do not lend themselves to computing $\operatorname{Ext}(H, A)$ in terms of $\operatorname{Ext}\left(H_{1}, A\right), \operatorname{Ext}\left(H / H_{1}, A\right), \cdots$ etc. Therefore, we assume that $H$ is connected, so that every extension of $A$ by $H$ is central. Let $\omega: L \rightarrow H$ be a universal covering for $H$ with kernel $K$. Then $K$ is a central subgroup, so from Proposition 2.2.2, with the help of Theorem 4.5, we have an exact sequence

$\operatorname{Hom}(L, A) \longrightarrow \operatorname{Hom}(K, A) \longrightarrow \operatorname{Ext}(H, A) \longrightarrow(0)$. 
Let $\operatorname{Hom}(L, A)_{K}$ denote the image of $\operatorname{Hom}(L, A)$ in $\operatorname{Hom}(K, A)$, i.e., the group of all rational homomorphisms $K \rightarrow A$ which extend to rational homomorphisms $L \rightarrow A$. We have the following proposition.

Proposition 5.4. Let $A$ and $H$ be reductive pro-affine algebraic groups over $F$ with $A$ abelian and $H$ connected. Let $\omega: L \rightarrow H$ be a universal covering of $H$ with kernel $K$. Then $\operatorname{Ext}(H, A) \cong$ $\operatorname{Hom}(K, A) / \operatorname{Hom}(L, A)_{K}$.

Suppose in the above proposition that $H$ is semisimple, i.e., that $C_{1}(H)$ is trivial. Then $L$ is semisimple as well, so $\operatorname{Hom}(L, A)=(0)$ because $A$ is abelian and $L$ is equal to the algebraic hull of its commutator subgroup. This gives the following corollary.

CoROllary 5.5. If $A$ and $H$ are pro-affine algebraic groups over $F$ with $A$ reductive and abelian and $H$ semisimple, then $\operatorname{Ext}(H, A) \cong \operatorname{Hom}(K, A)$ where $K$ is the fundamental group of $H$, i.e., the kernel of a universal covering of $H$.

Now suppose, in Proposition 5.4, that $A$ is equal to a product $\Pi_{s} F^{*}$ over some indexing set $S$ of copies of $F^{*}$. Then $A$ is injective in the category of reductive ablian pro-affine algebraic groups over $F$. Now $L=C_{1}(L) \times L^{\prime}$ as in Lemma 1.1, and we have $\omega\left(C_{1}(L)\right)=C_{1}(H)$ and $\omega\left(L^{\prime}\right)=H^{\prime}$. Put $D=C_{1}(H) \cap H^{\prime}$ and $M=$ $K \cap\left(1 \times L^{\prime}\right)$, so $M$ is the kernel of the restriction of $\omega$ to $L^{\prime}$, i.e., the fundamental group of $H^{\prime}$. The restriction homomorphism $\operatorname{Hom}(K, A) \rightarrow \operatorname{Hom}(M, A)$ is surjective because $A$ is injective in the category of reductive abelian groups. $L^{\prime}$, hence also $M$, is contained in the kernel of any rational homomorphism $L \rightarrow A$, so $\operatorname{Hom}(L, A)_{K}$ is contained in the kernel of the restriction homomorphism $\operatorname{Hom}(K, A) \rightarrow \operatorname{Hom}(M, A)$.

Now let $\uparrow: K \rightarrow A$ be a rational homomorphism whose restriction to $M$ is trivial. Put $E=C_{1}(L) \cap \omega^{-1}(D)$. Via the projection of $C_{1}(L) \times L^{\prime}$ onto the first factor, we obtain an isomorphism $K / M \rightarrow E$. So there exists a rational homomorphism $\phi: E \rightarrow A$ such that $\psi(x, y)=$ $\phi(x)$ for all $(x, y)$ in $K$. Now $\phi$ extends to a rational homomorphism $\bar{\phi}: C_{1}(L) \rightarrow A$ by the fact that $A$ is injective, as above. Define $\dot{\phi}^{\prime}: L=C_{1}(L) \times L^{\prime} \rightarrow A$ by $\phi^{\prime}(x, y)=\bar{\phi}(x)$ for all $(x, y)$ in $C_{1}(L) \times L^{\prime}$. The restriction of $\phi^{\prime}$ to $K$ coincides with $\psi$, so we have proved the following proposition.

Proposition 5.6. If $A$ is a product $\Pi_{s} F^{*}$ of copies of $F^{*}$ and $H$ is a connected reductive pro-affine algebraic group over $F$, then $\operatorname{Ext}(H, A) \cong \operatorname{Hom}(M, A)$ where $M$ is the fundamental group of $H^{\prime}$, 
the algebraic hull of the commutator subgroup of $H$. In particular, this holds if $A$ is a connected reductive abelian affine algebraic group over $F$.

\section{REFERENCES}

1. G. Hochschild, Algebraic groups and Hopf algebras, Illinois J. Math., 14 (1970), $52-65$.

2. - Algebraic automorphism groups, Illinois J. Math., 19 (1975), 131-144.

3. - Basic constructions in group extension theory, Contributions to Algebra, dedicated to Ellis Kolchin on the occasion of his 60th birthday, Academic Press, 1977.

4. - Coverings of pro-affine algebraic groups, Pacific J. Math., 35 (1970), 399-415.

5. - Introduction to Affine Glgebraic Aroups, Holden-Day, Inc. (1971).

6. G. Hochschild and G. D. Mostow, Pro-affine algebraic groups, Amer. J. Math., XCI (1969), 1127-1140.

7. - Representations and representative functions of Lie Groups, Annals of Math., 66 (1957), 495-542.

8. J. Humphreys, Linear Algebraic Groups, Springer-Verlag, New York (1975).

9. I. Kaplansky, Infinite Abelian Groups, U. of Michigan Press (1954).

Received July 14, 1977. This paper consists of the author's Ph. D. thesis (with a few minor revisions) submitted to the University of California, Berkeley, in 1976. I wish to express my heartfelt thanks to Gerhard Hochschild, whose direction was instrumental, and with whom working was a joy, while this research was being carried out.

RUTGERS UNIVERSITY

NEW BrunswiCK, NJ 08903 



\section{PACIFIC JOURNAL OF MATHEMATICS}

\section{EDITORS}

RICHARD ARENS (Managing Editor)

University of California

Los Angeles, California 90024

C. W. Curtis

University of Oregon

Eugene, OR 97403

C. C. MOORE

University of California

Berkeley, CA 94720

\section{J. DUGUNDJI}

Department of Mathematics University of Southern California Los Angeles, California 90007

R. Finn AND J. Milgram Stanford University Stanford, California 94305

\section{ASSOCIATE EDITORS}

E. F. BeCK ENBACH

B. H. NeUMaNN

F. WOLF

K. Yoshida

\section{SUPPORTING INSTITUTIONS}

UNIVERSITY OF BRITISH COLUMBIA CALIFORNIA INSTITUTE OF TECHNOLOGY UNIVERSITY OF CALIFORNIA MONTANA STATE UNIVERSITY UNIVERSITY OF NEVADA, RENO NEW MEXICO STATE UNIVERSITY OREGON STATE UNIVERSITY UNIVERSITY OF OREGON
UNIVERSITY OF SOUTHERN CALIFORNIA STANFORD UNIVERSITY UNIVERSITY OF HAWAII UNIVERSITY OF TOKYO UNIVERSITY OF UTAH WASHINGTON STATE UNIVERSITY UNIVERSITY OF WASHINGTON 


\section{Pacific Journal of Mathematics \\ Vol. 77, No. $1 \quad$ January, 1978}

Dan Amir, Chebyshev centers and uniform convexity ............... 1

Lawrence Wasson Baggett, Representations of the Mautner group. I ..... 7

George Benke, Trigonometric approximation theory in compact totally

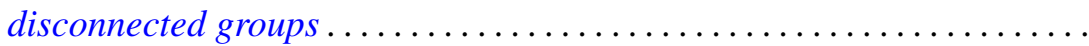

M. Bianchini, O. W. Paques and M. C. Zaine, On the strong compact-ported topology for spaces of holomorphic mappings ..................

Marilyn Breen, Sets with $(d-2)$-dimensional kernels

J. L. Brenner and Allen Kenneth Charnow, Free semigroups of $2 \times 2$

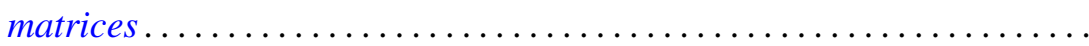

David Bressoud, A new family of partition identities .................

David Fleming Dawson, Summability of matrix transforms of stretchings

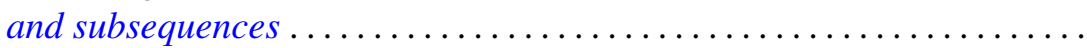

Harold George Diamond and Paul Erdôs, A measure of the nonmonotonicity

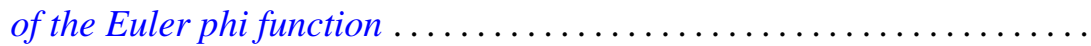

Gary Doyle Faulkner and Ronald Wesley Shonkwiler, Kernel dilation in reproducing kernel Hilbert space and its application to moment

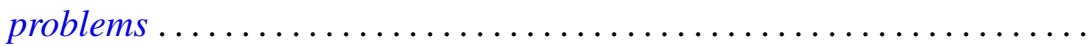

Jan Maksymilian Gronski, Classification of closed sets of attainability in the

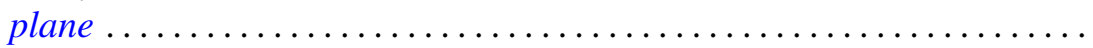

H. B. Hamilton and T. E. Nordahl, Semigroups whose lattice of congruences

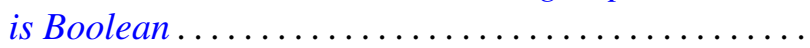

Harvey Bayard Keynes and D. Newton, Minimal $(G, \tau)$-extensions ...

Anthony To-Ming Lau, The Fourier-Stieltjes algebra of a topological

semigroup with involution.

B. C. Oltikar and Luis Ribes, On prosupersolvable groups ...

Brian Lee Peterson, Extensions of pro-affine algebraic groups ...

Thomas M. Phillips, Primitive extensions of Aronszajn spaces ...

Mehdi Radjabalipour, Equivalence of decomposable and 2-decomposable operators. .

M. Satyanarayana, Naturally totally ordered semigroups .

Fred Rex Sinal, A homeomorphism classification of wildly imbedded two-spheres in $S^{3}$

Hugh C. Williams, Some properties of a special set of recurring

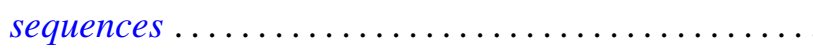

